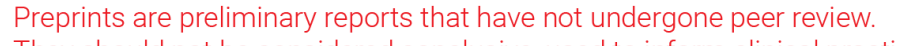 \\ They should not be considered conclusive, used to inform clinical practice, or referenced by the media as validated information. \\ Systematic analysis of JmjC gene family of Brassica napus and KDM5 subfamily involved in abiotic stress response
}

\section{Xinghui He}

Hunan Agricultural University

Jiao Pan

Hunan Agricultural University

Boyu Liu

Hunan Agricultural University

Chengfang Tan

Hunan Agricultural University

\section{Ying Ruan}

Hunan Agricultural University

\section{Yong Huang ( $\nabla$ yonghuang@hunau.edu.cn )}

Hunan Agricultural University https://orcid.org/0000-0001-9542-4807

\section{Research article}

Keywords: JmjC gene, evolution, histone lysine demethylases 5 subfamily, abiotic stress, Brassica napus

Posted Date: August 2nd, 2019

DOI: https://doi.org/10.21203/rs.2.11104/v1

License: () (1) This work is licensed under a Creative Commons Attribution 4.0 International License.

Read Full License 


\section{Abstract}

Background: Jumonji C (JmjC) proteins play an important role in plant development and stress response through the removal of lysine methylation from histones. Brassica napus, which originated from spontaneous hybridization between Brassica rapa and Brassica oleracea, is the most important oilseed crop after soybean, but evolutionary relationships and functions of JmjC proteins remain unclear. Results: $65 \mathrm{JmjC}$ genes were identified from B. napus genome, 29 from B. rapa, and 23 from B. oleracea. These genes were grouped into seven clades according to conserved sequences, and their catalytic activities of demethylation were predicted. Group-KDM4/JHDM3 for H3K4/9/27/36, Group-KDM5A/B for H3K4, Group-JmjC domain-only A/B for H3K27/36, Group-KDM3/JHDM2 for H3K9, and Group-JMJD6 may be for arginine demethylases. B. napus inherited most of its $\mathrm{JmjC}$ genes from its parents. The average retention rate of B. napus JmjC gene from B. rapa (93.1\%) and B. oleracea (82.6\%) exceeded that of all homologous gene pairs (83.7\%) across the whole B. napus genome. Thirteen new or duplicated $\mathrm{JmjC}$ genes have emerged in B. napus. Sequence similarity and domain organization analyses suggest that the functions of these genes might be diversified. Furthermore, KDM5 genes were examined under stress conditions due to H3K4 demethylation. Expression profiles indicated that the genes from B. napus are possibly involved in various stress responses. Conclusion: This study provides the first genome-wide characterization of $\mathrm{JmjC}$ genes in Brassica species. Its $\mathrm{JmjC}$ genes potentially have diverse functions, and its KDM5 genes might be involved in stress response. The results of this study facilitate the future functional characterization of the demethylation of JmjC family in Brassica crops.

\section{Background}

Epigenetics refers to heritable change for gene function that occurs without a change in DNA sequence and can dynamically regulate global gene expression through reversible chemical modifications on DNA and histones in eukaryotic chromatin [1]. Epigenetics regulation mainly includes acetylation, phosphorylation, histone methylation, DNA methylation, and small non-coding RNAs. Histone modification is an important epigenetics mechanism. Various post-translational covalent modifications, which primarily occur on histone ( $\mathrm{H} 3, \mathrm{H} 4, \mathrm{H} 2 \mathrm{~A}$, and $\mathrm{H} 2 \mathrm{~B})$ lysines and arginines residues, form "histone code" to regulate various biological processes [2]. Histone methylation is usually catalyzed by three protein families of histone methyltransferases: protein arginine methyltransferase family, Su (var)3-9, Enhancer-of-zeste and Trithorax (SET) domain family, and telomeric silencing disruptor that is also known as D0T1-Like (Kmt4/DOT1L) [3]. Histone lysine methylation, playing many different roles in biological processes ranging from heterochromatin formation to transcription regulation, is dynamically regulated by histone lysine methyltransferases (KMTs) and histone lysine demethylases (KDMs), and can be distinguished depending on the position of lysine residue and the number of added methyl groups in lysine residues, which carry mono-, di-, or tri-methylated groups [4].

As sessile organisms, plants are more susceptible to environmental factor than animals, though the former has developed complex mechanisms that perceive environmental stimuli and respond and adapt accordingly to the changing external environment. Histone modifications play important roles in a wide 
range of biological processes by affecting gene expression. For example, H3K4me3 and H3K36me3 are correlated to active gene expression, and H3K9me2 and H3K27me3 are associated to gene silencing [5]. The reversible epigenetic modifications dynamically regulate the plant's response to stress, including cold, freezing, saline, drought and submergence [6]. Abiotic stresses might induce various histone modifications to regulate the expression of genes with enhancing stress tolerance [2]. The active change in histone lysine methylation denotes a crucial role in plant adaptation to adverse environmental conditions. Athaliana genome-wide H3K4 methylation patterns (H3K4me1, H3K4me2 and H3K4me3) show dynamic responses to dehydration stress [7]. Arabidopsis ATX1, H3K4me3, is involved in dehydration response through ABA-dependent and -independent pathways [8]. Under drought stress, H3K4me3 enrichment is correlated with the activation of Arabidopsis drought stress-responsive genes, such as $R D 29 A$ and $R D 20[9,10]$. H3K4me3 can be maintained at low levels after rehydration and which could function as an epigenetic mark of drought stress memory [10]. Under heat stress, H3K4 methylation accumulates to induce gene expression and can be sustained after heat stress to positively respond on a future stress incident [11]. High-dose oxidative stress increases several histone methylation marks, including $\mathrm{H} 3 \mathrm{~K} 4 \mathrm{me} 3$, and low-dose oxidative stress increases the global levels of H3K4me3 and H3K27me3 [12]. Rice genome-wide H3K4me3 profiling showed that H3K4me3 modification is remarkably and positively correlated with the transcript level of drought stress-responsive genes [13]. Under submergence stress, the H3K4 methylation status of rice submergence-inducible $A D H 1$ and $P D C 1$ genes switches from dimethylation to trimethylation at the coding regions and is correlated with the increased expression of $A D H 1$ and $P D C 1$ [9].

Histone methylation is also a reversible process regulated by methylases and demethylases and might involve three distinct classes of demethylase. Petidylarginine deiminase 4 was identified as the first enzyme capable of antagonizing histone arginine methylation, but it cannot be strictly considered as a histone demethylase for its target citrulline [3]. KDMs mainly consist of LSD1/KDM1s (Lysine specific demethylase 1) and JmjC-domain enzymes, which both utilize oxidative mechanisms. LSD1, a flavindependent lysine specific demethylase member, was first reported as a histone lysine demethylase and transcriptional co-repressor specifically for histone $\mathrm{H} 3$ lysine 4 in humans [3]. Arabidopsis homologues of human LSD1 also exhibit similar demethylation and reduce H3K4 methylation in chromatin to promote floral transition [14].

JmjC, a highly conserved domain, was first reported by Takeuchi and colleagues in 1995 and was named as JmjC domain in $2000[15,16]$. This domain carries eight $\beta$-sheets forming enzymatically-active pocket with three conserved and necessary amino-acid residues for binding with $\mathrm{Fe}$ (II) cofactor and two additional residues for binding with a-ketoglutarate (aKG) $[17,18]$. Arabidopsis JmjC proteins are divided into five subfamilies: KDM4/JHDM3 (AtJMJ11-13), KDM5/JARID1 (AtJMJ14-19), JMJD6 (AtJMJ21/22), KDM3/JHDM2 (AtJMJ24-29) and JmjC domain-only (AtJMJ20 and AtJMJ30-32) [19]. The H3K4 methylases and demethylases dynamically balance the H3K4 methylation status among H3K4me1, H3K4me2 and H3K4me3, to maintain the optimum level of H3K4 methylation and adapt to external environment. KDM5 is a specific subfamily that specifically removes H3K4 methylation modifications. However, most reports on H3K4 demethylase functions were mainly focused on regulating plant 
development. For example, AtJMJ14/PKDM7B, a histone H3K4 demethylase, represses floral integrators Flowering Locus $T(F T), A P 1, S O C 1$ and $L F Y$ during vegetative growth [20, 21]. AtJMJ15 regulates flowering time by demethylating $\mathrm{H} 3 \mathrm{~K} 4 \mathrm{me} 3$ at Flowering Locus $C(F L C)$ chromatin [22]. AtJMJ18 is dominantly expressed in companion cells exhibiting H3K4me3 and H3K4me2 demethylase activity of FLC. atjmj18 mutation results in a weak late-flowering phenotype, and its overexpression induces earlyflowering [23]. Moreover, the overexpression of AtJMJ15 may regulate gene expression that enhances stress tolerance [24]. Although several functions of H3K4 methylation modifications in response to abiotic stresses have been reported, only a few were evaluated.

Allotetraploid species Brassica napus (oilseed rape, AACC, $2 n=38$ ) originated from interspecific spontaneous hybridization between Brassica rapa(AA, $2 n=20)$ and Brassica oleracea(CC, 2n=18) [21]. Brassica species might have diverged from a common ancestor with an Arabidopsis lineage from 14.520.4 million years ago [25]. The protein organization and function of JmjC domain in Brassica species and its relative relationship with model plant Arabidopsis remain uncharacterized. $B$. napus is currently the most important oilseed crop, preceded only by soybean. However, $B$. napus is vulnerable to abiotic stress that limits its growth and productivity and reduces its economic benefits. KDM5/JARID1 subfamily may regulate many abiotic stress responses genes through $\mathrm{H} 3 \mathrm{~K} 4 \mathrm{me} 3$ and $\mathrm{H} 3 \mathrm{~K} 4 \mathrm{me} 2$ downregulation but the roles of H3K4 demethylation in abiotic stress remain unknown.

\section{Results}

Chromosome maps of JmjC genes in Brassica

In this study, 21 Arabidopsis JmjC proteins were used as queries to Blastp in Brassica genomics (http://brassicadb.org/brad/). B. napus carried $65 \mathrm{JmjC}$ genes, whereas its parents $B$. rapa and $B$. oleracea had29 and 23, respectively(Additional file 1). $57 \mathrm{JmjC}$ genes of $B$. napus were mapped on 19 chromosomes (AACC, $2 n=38$ ), and 8 genes were still on scaffolds, in which 5 were from C-genomics and 2 from A-genomics. In addition, 23 B. oleracea genes and 29 B. rapa genes lactated on C01-C09 and A01A10 chromosomes, respectively.

The JmjC genes in $A$ and $C$ subgenomes of $B$. napus show nearly identical distributions to its ancestor genomes B. rapa (A-genome, 29) and B. oleracea (C-genome, 23) (Fig. 1). A02 and A07 chromosomes only exist in one member of $B$. napus, which is similar to its ancestor $B$. rapa genomes. A09 chromosome carries the highest number, seven genes. Four tandem JmjC genes pairs located on chromosomes $A 03$, A09, and $\mathrm{C} 03$ in B. rapa (Fig. 1). The tandem duplicated genes BnJMJ27;e and BnJMJ27; fon A03 subgenome are derived from $B r J M J 27 ; a$ and $B r J M J 27 ; b$, which belong to AtJMJ27 orthology. Tandem duplicated gene pairs $B n J M J 27 ; d / B n J M J 27 ; b$ and $B n J M J 17 ; a / B n J M J 17 ; c$ might have resulted from the forming processes allotetraploidy of B. napus. However, BnJMJ27;a and BnJMJ27;g of C03 sub-genome are absent from the ancestor $B$. oleracea genomes, and the orthologous genes of these tandemly duplicated genes appear in the corresponding location of A03 subgenome, which indicate that $B n J M J 27 ; a$ and $B n J M J 27 ; g$ might have derived from the cross duplication of A03 subgenome. 
$B n J M J 31 ; a$ of $C 03$ subgenome, BnJMJ18;c of C08 subgenome, BnJMJ17;b, and BnJMJ29;c of C09 subgenome may have similar origins.

Phylogenetic analysis of $\mathrm{Jmj} C$ proteins in $B$. napus

A phylogenetic tree was constructed from $21 \mathrm{JmjC}$ proteins form Arabidopsis, 19 from O. sativa, 29 from B. rapa, 23 from B. oleracea, and 65 from B. napus to examine the evolutionary relationships of JmjC proteins. The proteins were renamed using lysine demethylases nomenclature by Chromdb database (http://www.chromdb.org/). The JmjC proteins of Brassica were divided into seven clades, except BoJMJ19;C and BoJMJ19;d: KDM4/JHDM3, KDM5, JmjC domain only A/B, JMJD6 and KDM3/JHDM2 groups. This classification pattern was similar to the one previously reported for JmjC-domain proteins in the green lineage [26]. JmjC, Jumonji $\mathrm{N}(\mathrm{JmjN})$ and zinc-finger (ZnF) motifs were the special motifs for KDM4/JHDM3; JmjC, JmjN, F/Y-rich N terminus (FYRN) and F/Y-rich C terminus (FYRC) for KDM5A; JmjC, JmjN and plant homeodomain (PHD) for KDM5B; JmjC and F-box for JMJD6; JmjC and RING (really interesting new gene) for KDM3/JHDM2; and JmjC domain for JmjC domain only A/B (Figs. 3-7). JmjN domain specifically exists in all proteins of KDM4/JHDM3, KDM5A and KDM5B group, except for BrJMJ14;a, BnJMJ16;b, BrJMJ17 and BnJMJ17;c. B. rapa, Arabidopsis and O. sativa possess similar amounts of JmjC proteins in KDM5B, JmjC domain-only A, JMJD6 and JmjC domain-only B group. However, B. oleracea does not have JmjC protein of KDM5B and JmjC domain-only A. B. napus has 63 JmjC proteins, which is more than the sum of those for $B$. oleracea and $B$. rapa (Fig. 2; Additional file 1). The gene pairs imply the closest relatives within the phylogenetic tree. JmjC phylogenetic tree identified 39 sister pairs consisting of $22 \mathrm{An}-\mathrm{Ar}$ and $17 \mathrm{Cn}$-Co (Fig. 2). Moreover, most of the sister pairs are also paralogous gene pairs between the An- and $\mathrm{Cn}$ - subgenomes. JmjC genes show partial evolution but are still conserved on the whole genome of Brassica.

\section{Group KDM4/JHDM3}

Group-KDM4/JHDM3 contains nine JmjC proteins from B. napus, four from B. rapa, five from B. oleracea, five from $O$. sativa and three from Arabidopsis (Fig. 3). Group-KDM4/JHDM3 can be divided into two subgroups according to phylogenetic relationship, domain characteristic and gene structure: subgroup-I with eight Brassica members and two Arabidopsis homologous genes, AtJMJ11 and AtJMJ12.

The domain organization of subgroup-I members show highly-conserved and shared JmjC, JmjN and ZnF domain. Subgroup-II contains 10 Brassica members and Arabidopsis homologous gene AtJMJ13 shared JmjC, and JmjN (Figs. 3A and 3B). KDM4 subfamily shares the JmjN and JmjC motifs. JmjN domain is the second highly-conserved domain that is close to the $\mathrm{N}$ terminus and shorter than JmjC domain [16]. The four tandem array ZnF domain of RELATIVE OF EARLY FLOWERING 6 (REF6)/AtJMJ12 targets motif CTCTGYTY, and the ZnF domain only exists in subgroup-I [27]. REF6 also tends to bind to hypo-methylated CTCTGYTY motifs in vivo [28]. Subgroup-I generally harbors 7-8 exons, but subgroup-II keeps highly similar gene structures with 10 exons (Fig. 3C). 
JmjC proteins have been discovered as Fe(II)- and aKG-dependent histone demethylases [17, 18]. The $\mathrm{JmjN}$ and JmjC domains, two non-adjacent domains, interact with each other through two ß-sheets and form a single functional unit to ensure the stability and appropriate transcription activity of Gis 1 and maintain the overall protein levels and function of Jhd2 H3K4-specific demethylase in budding yeast [29, 30]. KDM4/JHDM3 has conserved Fe(II) binding site (His and Gluresidues) and aKG binding site (Phe and Lysresidues) (Fig. 3D).

\section{Group-KDM5A/B}

KDM5/JARID1 further be divided into two groups: KDM5A and KDM5B (Fig. 2; Additional file 2). GroupKDM5A contains $18 \mathrm{JmjC}$ proteins from $B$. napus, 2 from $O$. sativa, 10 from $B$. rapa, 7 from $B$. oleracea and 5 from Arabidopsis (Fig. 4A). Group-KDM5B only has 4 JmjC proteins from Brassica and 1 from Arabidopsis homologous gene AtJMJ17. B. oleracea does not have KDM5B JmjC proteins, and B. napus carries 3 members.

Group-KDM5A is distinguished by JmjC, JmjN, FYRN and FYRC motifs (Fig. 4B). FYRN and FYRC are present in many chromatin-associated proteins associated with histone H3K4 methyltransferases [31]. The FYRN and FYRC domains of JMJ14 interact with NAC050 and NAC052 transcription factors to facilitate H3K4 demethylase recruitment [32]. Group-KDM5A group can be further divided into three subgroups: subgroup-I with 18 Brassica members and 3 Arabidopsis homologous genes, AtJMJ14, AtJMJ15 and AtJMJ18. These members show highly-conserved domain organization sharing JmjC, JmjN, FYRN, and FYRC domains, except BrJMJ14;a. The phylogenetic tree showed that $B$. oleracea does not have AtJMJ18 homologues. Moreover, BrJMJ18;b is clustered with BnJMJ18;a and BnJMJ18; $b$, as well as BrJMJ18; a with BnJMJ18;C and BnJMJ18;d (Fig. 4A). However, $B$. napus does not have a gene clustered with BrJMJ14;b, BoJMJ15;b, and BrJMJ15;b. Subgroup-II has seven Brassica members and Arabidopsis homologous gene AtJMJ16 sharing JmjC, FYRN, JmjN and FYRC domains, except $B n J M J 16$;e, which display highly-conserved domain organization, in addition to BrJMJ16;a with additional helicase superfamily C-terminal and DEAD-like helicases superfamily domains. Subgroup-III has 10 Brassica members and Arabidopsis homologous genes AtJMJ19 and share JmjC and JmjN domains, besides BnJMJ19;a and BnJMJ19;b with an additional transmembrane domain. This finding suggested that subgroup-III may have a relatively stable inheritance during the evolutionary process of allotetraploidy.

Group-KDM5B differs from group-KDM5A group in domain organization, which has BRIGHT and PHD but lacking FYRN and FYRC (Fig. 4F). BRIGHT is associated with H3K4 demethylase by DNA binding motif (CCGCCC) to regulate transcription [33]. PHD mainly exerts epigenetic effectors capable of recognizing or "reading" post-translational histone modifications and unmodified histone tails [34]. The original PHD role in gene transcription is acted as a reader of H3K4me3 in 2006 [35]. Many sophisticated functions of PHD were also determined, including $\mathrm{H} 3 \mathrm{~K} 9 \mathrm{me} 3$ recognition and binding to the N-terminus of $\mathrm{H} 3$, indicating its key roles in regulating transcription and chromatin structure [36]. All members of groupKDM5B group have BRIGHT or PHD domains (Fig. 4F), indicating their involvement in demethylation 
using JmjC domain associated with BRIGHT and PHD domains. The tandem duplication genes of $B n J M J 17 ; a$ and BnJMJ17;C (An 09) are derived from the ancestor gene BrJMJ17(Ar 09), but BnJMJ17;C is clustered with BnJMJ17;b (Cn 09) (Fig. 4E; Additional file 3).

Group-KDM5A/B shows a wide range intron/exon number (5-36), but sister gene pairs are relatively conserved in gene structure (Fig. 4). In group-KDM5A, subgroups-I/ $/$ are highly conserved in $\mathrm{Fe}(\mathrm{II})$ and aKG binding sites, except for BrJMJ16;b in which Phe is replaced by Met in aKG binding site, and His is replaced by $\mathrm{Arg} \mathrm{Fe}(\mathrm{II})$ binding site. In subgroup-III, Phe is replaced by GIn in aKG binding site, and BoJMJ19;C/d is variable in other Fe(II) and aKG binding sites (Fig. 4D). In group-KDM5B, BnJMJ17a gene structure is similar to its parent BrJMJ17 (Fig. 4G). Group-KDM5B is highly conserved in Fe(II) and aKG binding sites, similar to KDM4/JHDM3 group (Fig. 4H).

Group-JmjC domain-only A/B

Group-JmjC domain-only A/B and JMJD6 are distributed in different branches of a large clade. GroupJmjC domain-only A is close to group-JMJD6 but far from group-JmjC domain-only B. Group-JmjC domain-only $A$ and $B$ have same domain organization and only exist in JmjC domain (Fig. 2).

Group-JmjC domain-only A possesses the least number of JmjC proteins among the groups (Figs. 2 and 5A) and contains three Brassica members and one Arabidopsis homologous gene AtJMJ20. B. oleraace is lack of Group-JmjC domain-only A JmjC proteins (Fig. 5). BnJMJ20;b shares coincident gene structures, domain organizations and chromosomal map with BrJMJ2O (Figs. 1, 5B and 5C) indicating that the former may have originated from the latter. Chromosomal map, CDS cover and protein ID reveal that BnJMJ20;a might be the duplicate of BnJMJ20;b (Additional file 2).

JmjC domain-only B contains $17 \mathrm{JmjC}$ proteins: 6 from B. napus, 2 from B. oleracea, 1 from B. rapa, 1 from $O$. sativa and 3 from Arabidopsis. Group-JmjC domain-only B can be further divided into three subgroups. Subgroup-I contains four Brassica members and Arabidopsis homologous gene AtJMJ30 (Fig. 5E). Subgroup-II contains three Brassica members and Arabidopsis homologous gene AtJMJ31. Subgroup-III contains four Brassica members and Arabidopsis homologous gene AtJMJ32 (Fig. 5E). Subgroups-I and III show high conservation during the forming process of allotetraploid. $B$. napus perfect inherited JmjC genes from its parents B. oleracea and B.rapa:BnJMJ30;b originating from BoJMJ30;a and $B n J M J 30 ; a$ from BrJMJ30;a within subgroup-l; BnJMJ32;b originating from BoJMJ32;a and BnJMJ32; a from BrJMJ32 in subgroup-III. B. oleracea lacks JmjC proteins in subgroup-II. BnJMJ31;a exhibits notable similarity with BnJMJ31;b in terms of domain component and gene structure, indicating that $\mathrm{BnJMJ} 31$; a may have originated from the inserted duplicate of BnJMJ31;b belonged to paralogues gene (Figs. 5F and 5G).

Group-JmjC domain-only A has stable exon distribution harboring approximately 7-9 exons. Sequence alignment and logos analysis of $\mathrm{JmjC}$ domain reveal that $\mathrm{JmjC}$ domain-only A group is highly conserved in Fe(II) and aKG binding sites. However, compared with that in the KDM4/JHDM3 group, Phe is replaced by Thr in Fe(II) binding site (Fig. 5D). In group-JmjC domain-only B, subgroup-I genes contains 6 exons, 
subgroup- III harbors 4 exons, and subgroup- II has many exons (Fig. 5G). As compared with that in the KDM4/JHDM3 group, the Phe residue is replaced by Ser within AtJMJ31 orthology (Fig. 5H).

Group-JMJD6

The phylogenetic tree showed that the JMJD6 group is close to JmjC domain-only A group and includes five JmjC proteins from $B$. napus, three from $B$. oleracea, three from Arabidopsis, two from $B$. rapa and two from $O$. sativa. Each JmjC gene of $B$. napus is clustered with a corresponding homologous gene from B. oleracea or B. rapa (Figs. 1 and $6 \mathrm{~A}$ ).

On the basis of phylogenetic tree analysis and schematic diagrams, group-JMJD6 can further be divided into two subgroups (Fig. 6). Subgroup-I contains six Brassica members and Arabidopsis homologous gene AtJMJ21 having only JmjC domain, besides BoJMJ21;b protein with an additional F-box domain (Figs. 6A and 6B). Subgroup-Il contains four Brassica members and AtJMJ22 sharing JmjC and F-box domains, except AtJMJ22 missing F-box domain. However, their gene structure shows high conservation (Fig. 6). F-box domain recognizes a wide array of substrates and regulates many important biological processes by degrading cellular proteins in plants [37].

Subgroup-I generally harbors 15-16 exons, except BoJMJ21;a (4 exon) and BnJMJ21;a (9 exon). Subgroup-II keeps highly similar gene structures with 2-3 exons (Fig. 6C). Compared with that in KDM4/JHDM3 group, Phe is replaced by Ala within AtJMJ21 orthology and by Ser within AtJMJ22 orthology in JMJD6 (Fig. 6D).

Group-KDM3/JHDM2

The KDM3 \& JHDM2 group is the largest group with 48 JmjC proteins: 6 from Arabidopsis (JMJ24-29), 22 from B. napus, 8 from B. rapa, 5 from 0 . sativa, and 6 from B. oleracea (Figs. 2 and 7A). Group-KDM3 \& JHDM2 can be divided into four subgroups: subgroup-I containing 14 Brassica members and 3 Arabidopsis homologous genes, AtJMJ25, AtJMJ26 and AtJMJ29. These proteins have AT-hook motif, RING and DM domains, except JmjC domain. Subgroups-II/III/IV contain AtJMJ27, AtJMJ24 and AtJMJ28 and their homologue genes, respectively. Subgroups-III/IV show highly-conserved and shared JmjC and RING domains, except BoJMJ24;a (Fig. 7B). Moreover, their gene structure also shows corresponding conservation (Fig. 7C).

In group-KDM3/JHDM2 (Fig. 7B), half of the member harbors RING domain as the second primary domain. Cys- $X_{2}-$ Cys- $X_{(9-39)}$-Cys- $X_{(1-3)}{ }^{-H i s-} X_{(2-3)}{ }^{-C y s}-X_{2}-$ Cys $-X_{(4-48)}-$ Cys $-X_{2}-$ Cys is the canonical RING [38]. The RING domain of many proteins mainly binds to ubiquitin-conjugating enzymes and mediates the direct transfer of ubiquitin to substrate [38]. The AT-hook is a small DNA-binding motif with a preference for $A / T$ rich regions found in various proteins, such as the high mobility group proteins [39].

Sequence alignment and logos analysis of the JmjC domain reveal that subgroups-I and II are highly conserved in Fe(II) binding sites (His, Asp and Cys) and aKG binding sites (Thr and Lys), except 
BoJMJ29;a. Moreover, both sites of subgroup-IV are different: the His and Asp residues of Fe(II) binding sites are replaced by Gly and Glu residues, and the Thr of aKG binding sites is replaced by Lys residue (Fig. 7D). However, subgroup-III does not present conservation.

KDM5 expression in abiotic stress

Arabidopsis KDM5 genes play central roles in stress-responsive gene expression and gene priming by H3K4me3 demethylation [40]. The expression of genes related to the response for drought, high temperature and saline stresses was determined to characterize the corresponding function of KDM5 group homologues in B. napus abiotic stress response.

The expression profiles of JmjC genes in $B$. napus leaves at three different stress conditions were detected by real-time PCR (Fig. 10). BnJMJ16;a, BnJMJ17;b/c and BnJMJ18; a showed remarkably elevated expression under salt, drought and high temperature. However, BrJMJ19;a/c did not show significant expression changes and thus might not be involved in the stress response or are less important. The vast majority of $\mathrm{JmjC}$ genes showed remarkably elevated expression under drought treatment, except for BnJMJ14; $a$ and BnJMJ19;a/c/e. Most of the genes had higher expression under drought 5 or/and 10 day than under drought 15, except for BnJMJ17;c and BnJMJ19;b (Fig. 10A). However, only 6 (BnJMJ16;a, BnJMJ17, BnJMJ18;a and BnJMJ19;e) out of the $20 \mathrm{JmjC}$ genes showed elevated expression under high temperature treatment. BnJMJ16;a, BnJMJ17a/b and BnJMJ18;a expression was induced under 12 hours of high temperature treatment, but BnJMJ17;c and BnJMJ19;e were not substantially expressed until 36 hours (Fig. 10B). Moreover, nearly half of the JmjC genes (BnJMJ14, BnJMJ15;a, BnJMJ16;a, BnJMJ17;b/c and BnJMJ18;a/d) showed remarkable expression under $100 \mathrm{Mm} \mathrm{NaCl}$ treatment, besides BnJMJ15;c that was strongly induced by $200 \mathrm{mM} \mathrm{NaCl}$ stress ( Fig. 10C).

\section{Discussion}

JmjC genes evolution of $B$. napus

Brassica species may diverge from a common ancestor with the Arabidopsis lineage from 14.5-20.4 million years ago [25]. Arabidopsis JmjC genes only possess one orthologues gene in the B. rapa and $B$. oleracea genomes, except for the six and four Arabidopsis JmjC genes with two orthologues genes in $B$. rapa and $B$. oleracea genomes, respectively. In addition, most of the genes originated from KDM5A subfamily. Only one Arabidopsis JmjC genes had three orthologues genes in $B$. rapaand $B$. oleracea genomes. Six Arabidopsis JmjC genes lack orthologues in B. oleracea (Fig. 1; Additional file 2).

B. napus (AACC, $2 \mathrm{n}=38$ ) is an amphidiploid species derived from interspecific hybridization between two diploid progenitors, $B$. rapa $(A A, 2 n=20)$ and $B$. oleracea $(C C, 2 n=18)$ [41]. Nuclear genomes have remained essentially unaltered since amphidiploid species formation [42]. Similarly, the JmjC protein family appears to be extremely conserved during $B$. napus formation. Compared with the progenitor genomes of B. rapa and B. oleracea, 27 (93.1\%) JmjC orthologous genes pairs between An subgenome 
and 19 (82.6\%) between $\mathrm{Cn}$ subgenome in B. napus were conserved (Fig. 2). The average retention rates from ancestor exceed the rate of all homologous gene pairs (83.7\%) across the whole $B$. napus genome [43]. Each member of $B$. rapa and $B$. oleracea can be paired to at least one homologue of $B$. napus, except for five members of KDM5A subfamily: BrJMJ14;b, BrJMJ15;b, BoJMJ15;b, BoJMJ19;c and BoJMJ19;d. This finding indicated their absence during allotetraploid formation (Fig. 4). Gene duplication expands genome content and changes gene function to ensure the optimal adaptability and evolution of plants [44]. The $65 \mathrm{JmjC}$ proteins from $B$. napus were more than the total number of proteins for $B$. rapa (29) and B. oleracea (23) (Addition file 1). According to the systematic analysis of JmjC proteins (Figs. 2-7), BrJMJ13;b, BrJMJ14; b, BoJMJ15; b, BoJMJ19;c/d and BoJMJ24;a might have been lost, and 13 new or duplicated JmjC gene have emerged in $B$. napus. Gene duplication events were confirmed by Yang et al. [45] and Sun et al. [46]. BnJMJ16;e/BnJMJ16;d, BnJMJ18;a/BnJMJ18;b, BnJMJ18;d/BnJMJ18;c, $B n J M J 31 ; a / B n J M J 31 ; b[B n J M J 29 ; b / B n J M J 29 ; d$ and $B n J M J 17 ; a / B n J M J 17 ; b$ duplicated genes pairs may have been derived from the existing JmjC gene from $B$. rapa and $B n J M J 28 ; a / B n J M J 28 ; b$ and $B n J M J 29 ; a / B n J M J 29 ; c$ pairs from $B$. oleracea (Addition file 1). These genes pairs were duplicated through segmental duplication (Addition file2). The parent of BnJMJ17; c was not found using the method by Yang et al. [45] and Sun et al. [46], but the JmjC domain sequence was consistent with the BnJMJ17;b.

\section{Conservation and function of JmjC proteins of B. napus}

Histone modification regulates plant development events by epigenetically silencing or activating target gene expression. Histone methylation is an important method to control plant development and stress response. In general, $\mathrm{H} 3 \mathrm{~K} 4 \mathrm{me} 2 / \mathrm{me} 3$ and $\mathrm{H} 3 \mathrm{~K} 36$ correlates with transcriptional activation, and $\mathrm{H} 3 \mathrm{~K} 9 \mathrm{me} 2$ and H3K27me3 correlates with gene silencing [5]. Histone modification status is regulated by KMTs and KDMs. KDMs can balance histone methylation through the removal of methylated residue of histone lysine or arginine. Histone demethylation, catalyzed by JmjC proteins, mainly occurs at the Lys residues of histone $\mathrm{H} 3(\mathrm{~K} 4, \mathrm{~K} 9, \mathrm{~K} 27$, and K36), which is involved in plant developmental stages [47], defense against [48,49], proteasomal degradation [50] and circadian clock [51]. In addition, JmjC protein regulates cell cycle progression by regulating H4K20 methylation [52]. Recent studies showed that arginine demethylation can also be catalyzed using a subset of JmjC KDMs [53].

JmjC domain proteins have been claded into seven groups [26]. KDM4/JHDM3 was involved in multidemethylation, e.g., AtJMJ11 contributes to the control of flowering time and other developmental processes by H3K27m3, H3K9me3 and H3K4me3 demethylation [54-57]. AtJMJ12/REF6 is involved in $\mathrm{H} 3 \mathrm{~K} 4 \mathrm{me} 2 / 3, \mathrm{H} 3 \mathrm{~K} 27 \mathrm{me} 2 / 3$ and H3K36me2/3 demethylation [27, 47, 58-61]. OsJM12;a/JMJ705 is involved in biotic stress-response through the $\mathrm{H} 3 \mathrm{~K} 27 \mathrm{me} 2 / 3$ demethylation of defense-related genes and increases basal and induced expression during pathogenic infection [62]. KDM5A might be involved in H3K4 demethylase activity, e.g., AtJMJ14 for histone H3K4 demethylation of $F T$ [20, 21], AtJMJ15 for histone $\mathrm{H} 3 \mathrm{~K} 4 \mathrm{me} 3$ demethylation of $F L C$ [22] and $\mathrm{H} 3 \mathrm{~K} 4 \mathrm{me} / 3$ demethylation for stress response genes [24] and AtJMJ18 for H3K4me2/3 demethylation for FLC [23]. JMJ703/OsJM16;a is also a histone H3K4 demethylase involved in transposon regulation and stem elongation [63, 64]. JMJ704/OsJM14;a exhibits 
$\mathrm{H} 3 \mathrm{~K} 4 \mathrm{me} 2 / 3$ demethylase activity and positively regulates rice defense response against Xanthomonas oryzae pv. oryzae [65]. The BRIGHT and PHD domains of KDM5B suggest its association with H3K4 demethylase [33-35]. JmjC domain-only A AtJMJ20 has a crucial role in removing histone arginine methylases at Gibberellin $3 \beta$-hydroxylase 1 (GA3Ox 1) and GA3ox2 and can positively regulate seed germination through demethylation increasing the gibberellic acid levels [66]. JmjC domain-only $B$ AtJMJ30 mediates plant responses to temperature and light to determine the timing of reproduction through $\mathrm{H} 3 \mathrm{~K} 36 \mathrm{me} 2$ demethylation of $F T$ [67] or H3K27me3 demethylation of $F L C$ [68]. JMJD6 AtJMJ22 acts as histone arginine demethylases and positively regulates seed germination with AtJMJ20 [66]. KDM3/JHDM2 IBM1/AtJMJ25 can negatively regulate DNA methylation, which prevents the ectopic accumulation of DNA methylation through the removal of heterochromatic H3K9 methylation mark [69]. AtJMJ27 modulates defense against pathogens and flowering time as H3K9 histone demethylase [48].

$65 \mathrm{JmjC}$ proteins of $B$. napus were clustered into seven groups based on phylogenetic and domain organization (Figs. 2-7; Supplement file 1), which displayed various histone demethylases activity. JmjC proteins of $B$. napus were an evolutionarily-conserved family during allotetraploid formation (Figs. 1 and 2). According to gene structure, domain origination, phylogenetic tree, and relationship with known functional proteins from model plant Arabidopsis, the substrate specifics of BnJmjC proteins can be inferred [26]. Group-KDM4/JHDM3 for demethylation of H3K4me2/3, H3K9me3, H3K27m2/3 and H3K36me2/3; group-KDM5A for demethylation of H3K4me2/3; group-KDM5B for demethylation of H3K4; group-JmjC domain-only A and group-JMJD6 for demethylation of arginine; group-JmjC domain-only B for demethylation of H3K36me2 and H3K27me3; group-KDM3/JHDM2, indirectly correlate with demethylation of $\mathrm{H} 3 \mathrm{~K} 9$.

KDM5 response to abiotic stress

Environmental conditions dramatically affect plant growth and crop production. Epigenetic mechanisms have also been involved in the regulation of stress response genes [70,71]. Santos et al. reviewed rice environmental epigenetic factors. Chromatin modifier enzymes may be transcriptionally regulated by abiotic stresses. The rice $\mathrm{H} 3 \mathrm{~K} 4 \mathrm{me} 3$ levels of $A D H 1$ and $P D C 1$ respond to submergence stress. $\mathrm{H} 3 \mathrm{~K} 4 \mathrm{me} 3$ is particularly correlated to gene expression regulation in dehydration stress [72]. Arabidopsis $\mathrm{H} 3 \mathrm{~K} 4 \mathrm{me} 3$ of $A H G 3$, catalyzed by ATX4 and ATX5, plays an essential role in drought stress response [73]. Arabidopsis $\mathrm{H} 3 \mathrm{~K} 4$ hypermethylation is associated with transcriptional activation and maintenance in heat stress response [74].

KDMs balance histone methylation level. KDM5 may be involved in H3K4 demethylase activity, for example, AtJMJ14 for histone H3K4 demethylation of $F T$ [20, 21], AtJMJ15 for histone H3K4me3 demethylation of $F L C$ [22] and $\mathrm{H} 3 \mathrm{~K} 4 \mathrm{me} 2 / 3$ demethylation for stress response genes [24] and AtJMJ18 for H3K4me2/3 demethylation of FLC [23]. Rice JMJ703/OsJM16;a is also a histone H3K4 demethylase involved in transposon activity regulation and stem elongation $[63,64]$. JMJ704/OsJM14;a exhibits H3K4me2/3 demethylase activity, which positively regulates defense response of rice against Xanthomonas oryzae pv. oryzae [65]. The phylogenetic analysis demonstrates that BnKDM5A members 
have conserved structural composition, and is almost consistent with Arabidopsis homologous genes indicating that $B$. napus members may also possess conserved function with H3K4 demethylation. Overexpression of Arabidopsis AtJMJ15 enhanced salt tolerance [24]. Group-KDM5/JARID1 may have H3K4 demethylase activity (Figs. 2 and 4). The much similar gene structures and domain organizations of JmjC genes between Arabidopsis and B. napus suggest their conserved biological functions (Fig. 4).

The expression patterns of BnKDM5 show its involvement in drought, high temperature and salt stress response (Fig. 8). KDM5B subgroup have three copies of $A t J M J 17(B n J M J 17 ; a / b / c)$. The similar gene structures and domain organizations of JmjC genes between Arabidopsis and B. napus suggest their conserved biological functions (Fig. 4). Under drought, high temperature or salt stress, all members of BnKDM5B exhibited remarkable elevated expression, except for BnJMJ17; $a$ under salt stress, suggesting that these homologous genes have similar responses to similar stress stimuli. Moreover, some members of BnKDM5B were relatively conserved between their homologous genes in certain stress. For instance, the homologous gene of AtJMJ15(BnJMJ15;a/b/c), AtJMJ16 (BnJMJ16;a/b/c/d), and AtJMJ18 $(B n J M J 18 ; a / b / c / d)$ showed similar stress response to drought stress with remarkable increased expression. However, their transcriptional responses to other stress stimuli may be different, even among homologous genes. For example, BnJMJ18;a shows remarkably elevated expression under high temperature without the homologous genes $B n J M J 18 ; b / c / d$ that indicates some of the homologous gene functions may differ. The expression profiling results present that BnKDM5A genes are relatively conserved and show remarkable response to identified stress. Moreover, most homologous genes of BnKDM4B genes demonstrated similar response to drought stress, but functions of some homologous

genes may have differed to adapt to various growth environments, which indicated that functions of BnKDM5 members is conserved and evolutional during allotetraploid formation.

\section{Methods}

Identification of JmjC proteins and chromosomal map construction

AtJMJ11-22 and AtJMJ24-32 JmjC protein sequences of Arabidopsis thaliana was obtained from official website of TAIR (http://www.arabidopsis.org/) and used as queries to BLASTp JmjC proteins of B. rapa, B. oleracea and B. napus in Brassica database (http://brassicadb.org/brad/blastPage.php). O. sativa were retrieved from Phytozome database (Version 12). The JmjC domain of candidate proteins was confirmed using SMART and NCBI, and proteins without JmjC domain were excluded. The JmjC gene loci information of 3 Brassica species were used to generate chromosome maps with the Mapchart 2.2 program [75].

Domain organization and phylogenetic analysis

The gene structures were visualized using the Gene Structure Display Server (http://gsds.cbi.pku.edu.cn/). The site information of the domain organization was used to construct a protein organization sketch map using DOG program [76]. 
Multiple sequence alignment of JmjC proteins ClustalW [77] and its resulting file were subjected to phylogenic analysis using MEGA7.0 program [78]. A tree was constructed based on the full-length protein sequences using neighbor-joining method with pairwise deletion and p-distance model, and a Bootstrap test of 1000 replicates for internal branch reliability. CDS sequences of JmjC were aligned with ClustalW (http://www.genome.jp/tools/clustalw/), and the resulting files were used to create Logo maps (http://weblogo.berkeley.edu/logo.cgi).

Duplicated JmjCgenes in B. napus

Duplicated genes were defined according to Yang et al [45] and Sun et al [46]. The full-length CDS sequence coverage and amino acid identities were determined using Blastn/Blastp at the NCBI website. The number of non-synonymous mutations (Ka) and the number of synonymous substitutions (Ks) of duplicated genes were calculated by DnaSP 6.0 [79]. The Ka/Ks ratios between duplicated genes and gene pairs were analyzed to determine the mode of selection. The duplication time ( $T$, million years ago, MYA) was calculated as $\mathrm{T}=\mathrm{Ks} / 2 \lambda \times 10^{-6}\left(\lambda=1.5 \times 10^{-8}\right)$ for $B$. napus [80].

Plant material and stress treatment

Brassica napus L. cv. Xiangyou 15 seedlings (The seeds were provided by Key Laboratory of Crop Epigenetic Regulation and Development in Hunan Province, Hunan Agricultural University, and Dr. Yong Huang identified the plant material) were grown in a growth chamber at $22^{\circ} \mathrm{C}$ chamber in a $16 \mathrm{~h}$ light $/ 8 \mathrm{~h}$ dark photoperiod. One-month old plants with 4 true leaves were treated. For drought stress, the seedlings were grown without watering, and leaves were sampled at $0,5,10$, and 15 days. For salt stress, seedlings were treated with $0,100,200$, and $300 \mathrm{mM} \mathrm{NaCl}$, and leaves were harvested 3 days after treatment. For high temperature stress, seedlings were grown in $40^{\circ} \mathrm{C}$, and leaves were harvested at $0,12,24$, and $36 \mathrm{~h}$ after treatment. All harvested samples were immediately frozen in liquid nitrogen. Three independent biological replicates for each treatment were conducted.

RNA extraction and real-time quantitative PCR (RT-qPCR)

RNA extraction and real-time quantitative RT-PCR were conducted as described previously [81]. Total RNA was extracted from samples using a TRIzol reagent kit (Invitrogen, Carlsbad, CA, US) following manufacturer's instructions. Total RNA were reverse transcribed into cDNA using Revert Aid RT Kit (Thermo Fisher, USA). The primer pairs used for real-time PCR were designed using Beacon Designer 8.20 according to the CDS sequences of the JmjC genes in B. nupas (Additional file 4), and were synthesized by Generay Biotech (Generay, PRC). The real-time quantitative PCR was conducted using Fast Start Universal SYBR Green Master (ROX) (Roche, Switzerland) on a CF x 96 Real Time System (BIORAD). Each sample was run in triplicate. The expression levels of group-KDM5 genes were calculated with the 2- $\triangle \triangle C T$ method [82].

\section{Abbreviations}


aKG: a-ketoglutarate; ELF6: EARLY FLOWERING 6; FLC: Flowering Locus C, FT: Flowering Locus T; FYRC: F/Y-rich C terminus; FYRN: F/Y-rich N terminus; JmjC: Jumonji C; JmjN: Jumonji N; KDMs: histone lysine demethylases; KMTs: histone lysine methyltransferases; PHD: plant homeodomain; ZnF: zinc-finger

\section{Declarations}

Ethics approval and consent to participate

The field trail experiments in the current study were permitted by the local government in China.

Consent for publication

Not applicable.

Competing interests

The authors declare that they have no competing interests.

Availability of data and material

All data generated or analyzed during this study are included in this published article and its Additional files. The datasets generated and analysed during the current study are available from the corresponding author on reasonable request.

Funding

This work was funded by Natural Science Foundation of Hunan Province (2019JJ40116), Scientific Research Project of Hunan Provincial Education Department (18K041), and Training Program for Youth Backbone Teacher in University of Hunan Province (Resistance and Epigenetic Mechanism of Rice and Brassica 2015). The funding sources had no influence on the design of the study and collection, analysis, and interpretation of data and in writing the manuscript.

Authors' contributions

$\mathrm{XH}$ contributed most of the experiments and drafted the manuscript; PJ, LB and TC contributed the sample and qRT-PCR; RY and HY conceived and directed the study; HY wrote the final version of the manuscript; All authors read and approved the final manuscript.

Acknowledgements

Not applicable.

\section{References}

1. He C, Cole P. Introduction. epigenetics. Chem Rev. 2015;115(6):2223-4. 
2. Bannister AJ, Kouzarides T. Regulation of chromatin by histone modifications. Cell Res. 2011;21(3):381-95.

3. Greer EL, Shi Y. Histone methylation. a dynamic mark in health, disease and inheritance. Nat Rev Genet. 2012;13(5):343-57.

4. Liu C, Lu F, Cui X, Cao X. Histone methylation in higher plants. Annu Rev Plant Biol.2010;61:395-420.

5. Huang Y, Liu CL, Shen WH, Ruan Y. Phylogenetic analysis and classification of the Brassica rapa SETdomain protein family. Bmc Plant Biol.2011;11:175.

6. Bej S, Basak J. Abiotic stress induced epigenetic modifications in plants: how much do we know? Plant epigenetics. Springer, Cham, 2017; 493-512.

7. van Dijk K, Ding Y, Malkaram S, Riethoven JJ, Liu R, Yang J, et al. Dynamic changes in genome-wide histone $\mathrm{H} 3$ lysine 4 methylation patterns in response to dehydration stress in Arabidopsis thaliana. BMC Plant Biol. 2010;10:238.

8. Ding Y, Avramova Z, Fromm M. The Arabidopsis trithorax-like factor ATX1 functions in dehydration stress responses via ABA-dependent and ABA-independent pathways. Plant J. 2011;66(5):735-74.

9. Qiao W, Fan L. Epigenetics, a mode for plants to respond to abiotic stresses. Front Biol (Beijing). 2011;6(6):477-81.

10. Kim JM, To TK, Ishida J, Matsui A, Kimura H, Seki M. Transition of chromatin status during the process of recovery from drought stress in Arabidopsis thaliana. Plant Cell Physiol. 2012;53(5):847-56.

11. Lamke J, Brzezinka K, Altmann S, Baurle I. A hit-and-run heat shock factor governs sustained histone methylation and transcriptional stress memory. EMBO J. 2016;35(2):162-75.

12. Niu Y, DesMarais TL, Tong Z, Yao Y, Costa M. Oxidative stress alters global histone modification and DNA methylation. Free Radic Biol Med. 2015;82:22-8.

13. Zong W, Zhong X, You J, Xiong L. Genome-wide profiling of histone H3K4-tri-methylation and gene expression in rice under drought stress. Plant Mol Biol. 2013;81(1-2):175-88.

14. Jiang D, Yang W, He Y, Amasino RM. Arabidopsis relatives of the human lysine-specific Demethylase1 repress the expression of FWA and FLOWERING LOCUS C and thus promote the floral transition. Plant Cell. 2007;19(10):2975-87.

15. Takeuchi T, Yamazaki Y, Katoh-Fukui Y, Tsuchiya R, Kondo S, Motoyama J, et al. Gene trap capture of a novel mouse gene, jumonji, required for neural tube formation. Genes Dev.1995;9(10):1211-22.

16. Balciunas D, Ronne H. Evidence of domain swapping within the jumonji family of transcription factors. Trends Biochem Sci. 2000;25(6):274-6. 
17. Klose RJ, Kallin EM, Zhang Y. JmjC-domain-containing proteins and histone demethylation. Nat Rev Genet. 2006;7(9):715-27.

18. Chen Z, Zang J, Whetstine J, Hong X, Davrazou F, Kutateladze TG, et al. Structural insights into histone demethylation by JMJD2 family members. Cell. 2006;125(4):691-702.

19. Luo M, Hung F-Y, Yang S, Liu X, Wu K. Histone lysine demethylases and their functions in plants. Plant Mol Biol Rep. 2013;32(2):558-65.

20. Lu F, Cui X, Zhang S, Liu C, Cao X. JMJ14 is an H3K4 demethylase regulating flowering time in Arabidopsis. Cell Res. 2010;20(3):387-90.

21. Yang W, Jiang D, Jiang J, He Y. A plant-specific histone H3 lysine 4 demethylase represses the floral transition in Arabidopsis. Plant J.2010;62(4):663-73.

22. Yang H, Mo H, Fan D, Cao Y, Cui S, Ma L. Overexpression of a histone H3K4 demethylase, JMJ15, accelerates flowering time in Arabidopsis. Plant Cell Rep. 2012;31(7):1297-308.

23. Yang $\mathrm{H}$, Han $\mathrm{Z}$, Cao Y, Fan D, Li H, Mo H, et al. A companion cell-dominant and developmentally regulated $\mathrm{H} 3 \mathrm{~K} 4$ demethylase controls flowering time in Arabidopsis via the repression of FLC expression. PLoS Genet. 2012;8(4):e1002664.

24. Shen Y, Conde ESN, Audonnet L, Servet C, Wei W, Zhou DX. Over-expression of histone H3K4 demethylase gene JMJ15 enhances salt tolerance in Arabidopsis. Front Plant Sci. 2014;5:290.

25. Yang YW, Lai KN, Tai PY, Li WH. Rates of nucleotide substitution in angiosperm mitochondrial DNA sequences and dates of divergence between Brassica and other angiosperm lineages. J Mol Evol.1999;48(5):597-604.

26. Huang Y, Chen D, Liu C, Shen W, Ruan Y. Evolution and conservation of JmjC domain proteins in the green lineage. Mol Genet Genomics.2016;291(1):33-49.

27. Li C, Gu L, Gao L, Chen C, Wei CQ, Qiu Q, et al. Concerted genomic targeting of H3K27 demethylase REF6 and chromatin-remodeling ATPase BRM in Arabidopsis. Nat Genet.2016; 48(6):687-93.

28. Qiu Q, Mei H, Deng X, He K, Wu B, Yao Q, et al. DNA methylation repels targeting of Arabidopsis REF6. Nature Commun. 2019;10(1):2063.

29. Huang F, Chandrasekharan MB, Chen YC, Bhaskara S, Hiebert SW, Sun ZW. The JmjN domain of Jhd2 is important for its protein stability, and the plant homeodomain (PHD) finger mediates its chromatin association independent of H3K4 methylation. J Biol Chem. 2010, 285(32):24548-61.

30. Quan Z, Oliver SG, Zhang N. JmjN interacts with JmjC to ensure selective proteolysis of Gis1 by the proteasome. Microbiology. 2011;157(Pt 9):2694-701. 
31. Garcia-Alai MM, Allen MD, Joerger AC, Bycroft M. The structure of the FYR domain of transforming growth factor beta regulator 1. Protein Sci. 2010;19(7):1432-8.

32. Zhang S, Zhou B, Kang Y, Cui X, Liu A, Deleris A, et al. C-terminal domains of a histone demethylase interact with a pair of transcription factors and mediate specific chromatin association. Cell Discov. 2015;1: pii: 15003.

33. Tu S, Teng YC, Yuan C, Wu YT, Chan MY, Cheng AN, et al. The ARID domain of the H3K4 demethylase RBP2 binds to a DNA CCGCCC motif. Nat Struct Mol Biol.2008;15(4):419-21.

34. Musselman CA, Kutateladze TG. Handpicking epigenetic marks with PHD fingers. Nucleic Acids Res.2011;39(21):9061-71.

35. Wysocka J, Swigut T, Xiao H, Milne TA, Kwon SY, Landry J, et al. A PHD finger of NURF couples histone H3 lysine 4 trimethylation with chromatin remodelling. Nature. 2006;442(7098):86-90.

36. Wang Q, Liu J, Wang Y, Zhao Y, Jiang H, Cheng B. Systematic analysis of the maize PHD-Finger gene family reveals a subfamily involved in abiotic stress response. Int J Mol Sci.2015;16(10):23517-44.

37. Gupta S, Garg V, Kant C, Bhatia S. Genome-wide survey and expression analysis of F-box genes in chickpea. BMC Genomics.2015;16:67.

38. Deshaies RJ, Joazeiro CA. RING domain E3 ubiquitin ligases. Annu Rev Biochem. 2009;78:399-434.

39. Klosterman SJ, Hadwiger LA. Plant HMG proteins bearing the AT-hook motif. Plant Sci.2002;162(6):855-66.

40. Jaskiewicz M, Conrath U, Peterhansel C. Chromatin modification acts as a memory for systemic acquired resistance in the plant stress response. EMBO Rep.2011;12(1):50-5.

41. Nagaharu U. Genome analysis in Brassica with special reference to the experimental formation of $B$. Napus and peculiar mode of fertilization. Jour Bot. 1935;7:389-452.

42. Parkin IA, Sharpe AG, Keith DJ, Lydiate DJ. Identification of the A and C genomes of amphidiploid Brassica napus (oilseed rape). Genome. 1995;38(6):1122-31.

43. Chalhoub B, Denoeud F, Liu S, Parkin IA, Tang H, Wang X, et al. Early allopolyploid evolution in the post-Neolithic Brassica napus oilseed genome. Science. 2014;345(6199):950-3.

44. Xu G, Guo C, Shan H, Kong H. Divergence of duplicate genes in exon-intron structure. Proc Natl Acad Sci USA. 2012;109(4):1187-92.

45. Yang S, Zhang X, Yue JX, Tian D, Chen JQ. Recent duplications dominate NBS-encoding gene expansion in two woody species. Mol Genet Genomics.2008;280(3):187-98. 
46. Sun R, Wang K, Guo T, Jones DC, Cobb J, Zhang B, et al. Genome-wide identification of auxin response factor (ARF) genes and its tissue-specific prominent expression in Gossypium raimondii. Funct Integr Genomics. 2015;15(4):481-93.

47. Cui X, Lu F, Qiu Q, Zhou B, Gu L, Zhang S, et al. REF6 recognizes a specific DNA sequence to demethylate H3K27me3 and regulate organ boundary formation in Arabidopsis. Nature Genetics.2016;48:694.

48. Dutta A, Choudhary P, Caruana J, Raina R. JMJ27, an Arabidopsis H3K9 histone demethylase, modulates defense against Pseudomonas syringae and flowering time. Plant J. 2017;91(6):1015-28.

49. Audonnet L, Shen Y, Zhou DX. JMJ24 antagonizes histone H3K9 demethylase IBM1/JMJ25 function and interacts with RNAi pathways for gene silencing. Gene Expr Patterns.2017;25-26:1-7.

50. Deng S, Jang IC, Su L, Xu J, Chua NH. JMJ24 targets CHROMOMETHYLASE3 for proteasomal degradation in Arabidopsis. Genes Dev.2016;30(3):251-6.

51. Lu SX, Knowles SM, Webb CJ, Celaya RB, Cha C, Siu JP, et al. The Jumonji C domain-containing protein JMJ30 regulates period length in the Arabidopsis circadian clock. Plant Physiol. 2011;155(2):90615.

52. Liu W, Tanasa B, Tyurina OV, Zhou TY, Gassmann R, Liu WT, et al. PHF8 mediates histone H4 lysine 20 demethylation events involved in cell cycle progression. Nature. 2010;466(7305):508-12.

53. Li S, Ali S, Duan X, Liu S, Du J, Liu C, et al. JMJD1B demethylates H4R3me2s and H3K9me2 to facilitate gene expression for development of hematopoietic stem and progenitor cells. Cell Rep.2018;23(2):389-403.

54. Noh B, Lee SH, Kim HJ, Yi G, Shin EA, Lee M, et al. Divergent roles of a pair of homologous jumonji/zinc-finger-class transcription factor proteins in the regulation of Arabidopsis flowering time. Plant Cell.2004;16(10):2601-13.

55. Bendahmane $\mathrm{M}$, Jeong J-H, Song $\mathrm{H}-\mathrm{R}$, Ko J-H, Jeong Y-M, Kwon YE, et al. Repression of FLOWERING LOCUS T chromatin by functionally redundant histone $\mathrm{H} 3$ lysine 4 demethylases in Arabidopsis. PLOS ONE. 2009;4(11) :e8033.

56. Crevillen P, Yang H, Cui X, Greeff C, Trick M, Qiu Q, et al. Epigenetic reprogramming that prevents transgenerational inheritance of the vernalized state. Nature. 2014;515(7528):587-90.

57. Yu X, Li L, Li L, Guo M, Chory J, Yin Y. Modulation of brassinosteroid-regulated gene expression by Jumonji domain-containing proteins ELF6 and REF6 in Arabidopsis. Proc Natl Acad Sci USA.2008;105(21):7618-23. 
58. Ko JH, Mitina I, Tamada Y, Hyun Y, Choi Y, Amasino RM, et al. Growth habit determination by the balance of histone methylation activities in Arabidopsis. EMBO J. 2010;29(18):3208-15.

59. Lu F, Cui X, Zhang S, Jenuwein T, Cao X. Arabidopsis REF6 is a histone H3 lysine 27 demethylase. Nat Genet.2011;43(7):715-9.

60. Hou X, Zhou J, Liu C, Liu L, Shen L, Yu H. Nuclear factor Y-mediated H3K27me3 demethylation of the SOC1 locus orchestrates flowering responses of Arabidopsis. Nat Commun. 2014;5:4601.

61. Hyun Y, Richter R, Vincent C, Martinez-Gallegos R, Porri A, Coupland G. Multi-layered regulation of SPL15 and cooperation with SOC1 integrate endogenous flowering pathways at the Arabidopsis shoot meristem. Dev Cell.2016;37(3):254-66.

62. Li T, Chen X, Zhong X, Zhao Y, Liu X, Zhou S, et al. Jumonji C domain protein JMJ705-mediated removal of histone $\mathrm{H} 3$ lysine 27 trimethylation is involved in defense-related gene activation in rice. Plant Cell. 2013;25(11):4725-36.

63. Cui X, Jin P, Cui X, Gu L, Lu Z, Xue Y, et al. Control of transposon activity by a histone H3K4 demethylase in rice. Proc Natl Acad Sci USA. 2013;110(5):1953-58.

64. Chen Q, Chen X, Wang Q, Zhang F, Lou Z, Zhang Q, et al. Structural basis of a histone H3 lysine 4 demethylase required for stem elongation in rice. PLoS Genet. 2013;9(1):e1003239.

65. Hou Y, Wang L, Wang L, Liu L, Li L, Sun L, et al. JMJ704 positively regulates rice defense response against Xanthomonas oryzae pv. oryzae infection via reducing H3K4me2/3 associated with negative disease resistance regulators. BMC Plant Biol. 2015;15:286.

66. Cho JN, Ryu JY, Jeong YM, Park J, Song JJ, Amasino RM, et al. Control of seed germination by lightinduced histone arginine demethylation activity. Dev Cell.2012;22(4):736-48.

67. Yan Y, Shen L, Chen Y, Bao S, Thong Z, Yu H. A MYB-domain protein EFM mediates flowering responses to environmental cues in Arabidopsis. Dev Cell.2014;30(4):437-48.

68. Gan ES, Xu Y, Wong JY, Goh JG, Sun B, Wee WY, et al. Jumonji demethylases moderate precocious flowering at elevated temperature via regulation of FLC in Arabidopsis. Nat Commun. 2014;5:5098.

69. Saze H, Sasaki T, Kakutani T. Negative regulation of DNA methylation in plants. Epigenetics. 2014;3(3):122-4.

70. Baulcombe DC, Dean C. Epigenetic regulation in plant responses to the environment. Cold Spring Harb Perspect Biol.2014;6(9):a019471.

71. Begcy K, Dresselhaus T. Epigenetic responses to abiotic stresses during reproductive development in cereals. Plant Reprod. 2018;31(4):343-55. 
72. Santos AP, Serra T, Figueiredo DD, Barros P, Lourenco T, Chander S, et al. Transcription regulation of abiotic stress responses in rice: a combined action of transcription factors and epigenetic mechanisms. OMICS. 2011;15(12):839-57.

73. Liu Y, Zhang A, Yin H, Meng Q, Yu X, Huang S, et al. Trithorax-group proteins ARABIDOPSIS TRITHORAX4 (ATX4) and ATX5 function in abscisic acid and dehydration stress responses. New Phytol.2018;217(4):1582-97.

74. Liu HC, Lamke J, Lin SY, Hung MJ, Liu KM, Charng YY, et al. Distinct heat shock factors and chromatin modifications mediate the organ-autonomous transcriptional memory of heat stress. Plant $\mathrm{J}$. 2018;95(3):401-13.

75. Voorrips RE. MapChart: software for the graphical presentation of linkage maps and QTLs. J Hered.2002;93(1):77-8.

76. Ren J, Wen L, Gao X, Jin C, Xue Y, Yao X. DOG 1.0: illustrator of protein domain structures. Cell Res.2009;19(2):271-3.

77. Thompson JD, Higgins DG, Gibson TJ. CLUSTAL W: improving the sensitivity of progressive multiple sequence alignment through sequence weighting, position-specific gap penalties and weight matrix choice. Nucleic Acids Res. 1994; 22(22):4673-80.

78. Tamura K, Stecher G, Peterson D, Filipski A, Kumar S. MEGA6: molecular evolutionary genetics analysis version 6.0. Mol Biol Evol. 2013;30(12):2725-9.

79. Town CD, Cheung F, Maiti R, Crabtree J, Haas BJ, Wortman JR, et al. Comparative genomics of Brassica oleracea and Arabidopsis thaliana reveal gene loss, fragmentation, and dispersal after polyploidy. Plant cell. 2006;18(6):1348-59.

80. Koch MA, Haubold B, Mitchell-Olds T. Comparative evolutionary analysis of chalcone synthase and alcohol dehydrogenase loci in Arabidopsis, Arabis, and related genera (Brassicaceae). Mol Biol Evol. 2000;17(10):1483-98.

81. Huang $Y$, Mo Y, Chen P, Yuan X, Meng F, Zhu S, et al. Identification of SET domain-containing proteins in Gossypium raimondii and their response to high temperature stress. Sci Rep. 2016; 6:32729.

82. Livak KJ, Schmittgen TD: Analysis of relative gene expression data using real-time quantitative PCR and the 2(-Delta Delta C(T)) Method. Methods. 2001; 25(4):402-8.

\section{Additional Material}

Additional file 1:Detailed information of JmjC genes in Brassica

Sequences and information of JmjC genes and proteins came from http://brassicadb.org/brad/ 
Additional file 2: Duplicated JmjC genes of $B$. rapa

Ka and Ks were calculated by DnaSP 6.0; Cover of CDS and Identify of protein was checked by NCBI.

Additional file 3: NJ phylogenetic tree of KDM5

$48 \mathrm{JmjC}$ proteins sequences (Arabidopsis 6, B. rapa11, Rice3, B.napus 21, B.oleracea 7) were aligned using ClustalW and the phylogenetic tree analysis was performed using MEGA 7.0. The tree was constructed with the following settings: Statistical Method as Neighbor-joining; Include Sites as Partial deletion option for total sequence analyses; Substitution Model: Poisson model; and Bootstrap test of 1000 replicates for internal branch reliability.

Additional file 4: Primers list of group-V in B. napus

Fig. 1 Chromosomal distribution of Brassica genes

Brassica genes (57 B. napus, 23 B. oleracea and 29 B. rapa) was mapped on chromosomes except eight scaffolds genes of B. napus: A. B. napus genes distribution of A-genomics, B. B. rapa genes distribution, C. B. napus genes distribution of C-genomics, D. B. oleracea genes distribution. The scale on the chromosome represents megabases (Mb).

Fig. 2 Phylogenetic tree of JmjC domain proteins

The Phylogenetic tree included $21 \mathrm{JmjC}$ domain-containing proteins form Arabidopsis thaliana, 19 from Oryza sativa, 29 from Brassica rapa, 23 from Brassica oleracea and 65 from Brassica napus. The JmjC domain proteins can be grouped into7 groups based on the phylogenetic tree and domain organization. Different colors show different groups. JmjC domain protein sequences were aligned using ClustalW, and the phylogenetic tree analysis was performed using MEGA7.0.The trees were constructed with the following settings: tree inference as neighbor-joining; include sites as pairwise deletion option for total sequences analysis; substitution model as p-distance.

Fig. 3 The schematic diagrams of Group-KDM4/JHDM3

A. Phylogeny tree, B. domain organization, C. Gene structure, D. Logos analysis of JmjC domain.

Fig. 4 The schematic diagrams of Group-KDM5A/B

A/E. Phylogeny tree, B/F. domain organization, C/G. Gene structure, D/H. Logos analysis of JmjC domain.

Fig. 5 The schematic diagrams of Group-JmjC domain-onlyA/B

A/E. Phylogeny tree, B/F. domain organization, C/G. Gene structure, D/H. Logos analysis of JmjC domain.

Fig. 6 The schematic diagrams of Group-JMJD6 
A. Phylogeny tree, B. domain organization, C. Gene structure, D. Logos analysis of JmjC domain.

Fig. 7 The schematic diagrams of Group- KDM3\&JHDM2

A. Phylogeny tree, B. domain organization, C. Gene structure, D. Logos analysis of JmjC domain.

Fig. 8 Expression of B. naups KDM5 subfamily in response to drought, high temperature or $\mathrm{NaCl}$ stresses

Many of BnJMJ14-19 genes involved in drought, high temperature or $\mathrm{NaCl}$ stress response. The error bars depict SD, an asterisk represent corresponding gene significantly up- or down-regulated by Student's t test between the treatment and the control $(0.01<P<0.05)$, two represent $(p<0.01)$.

\section{Figures}



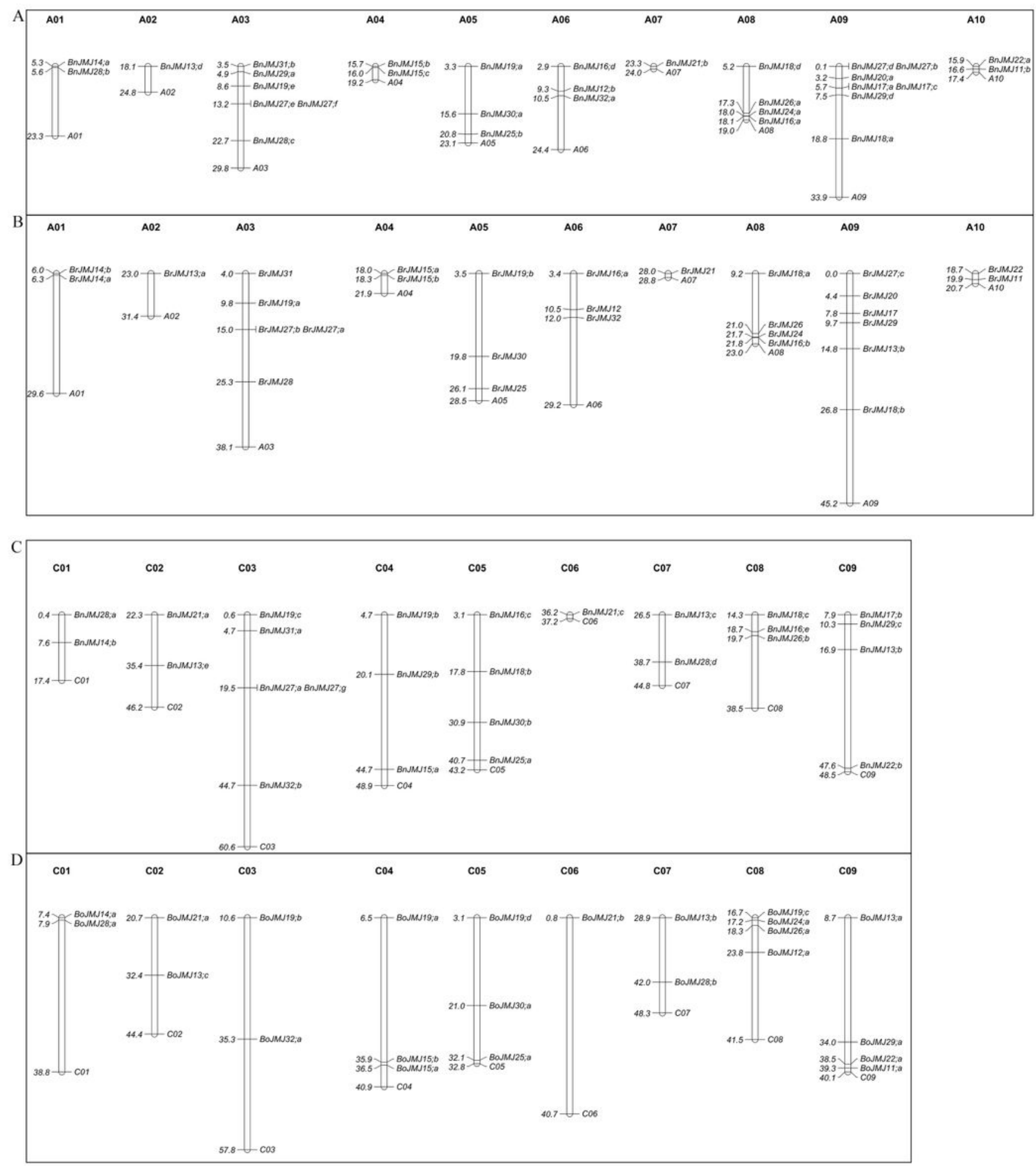

Figure 1

Chromosomal distribution of Brassica genes Brassica genes (57 B. napus, 23 B. oleracea and 29 B. rapa) was mapped on chromosomes except eight scaffolds genes of B. napus: A. B. napus genes distribution of A-genomics, B. B. rapa genes distribution, C. B. napus genes distribution of C-genomics, D. B. oleracea genes distribution. The scale on the chromosome represents megabases (Mb). 


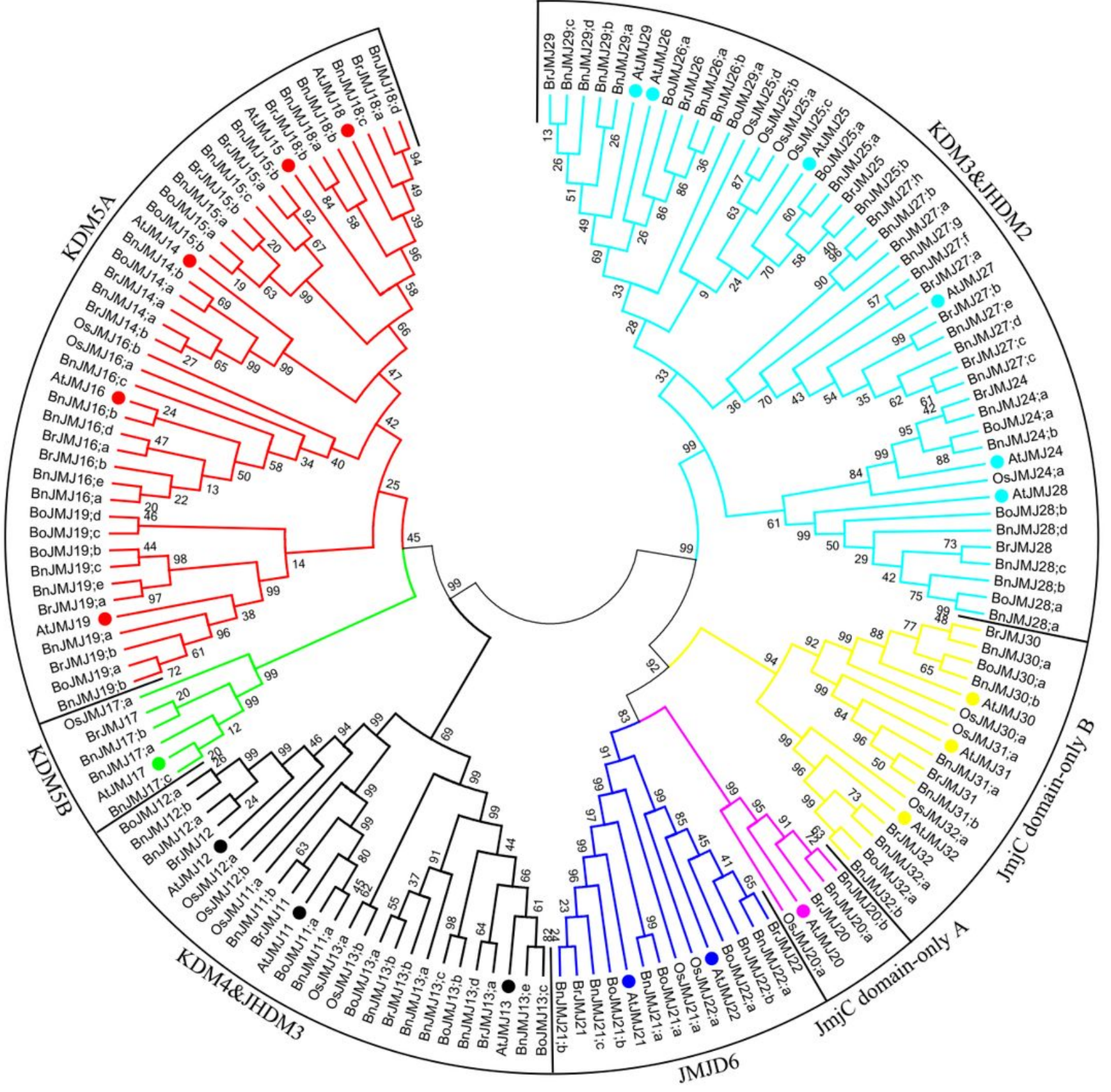

\section{Figure 2}

Phylogenetic tree of JmjC domain proteins The Phylogenetic tree included $21 \mathrm{JmjC}$ domain-containing proteins form Arabidopsis thaliana, 19 from Oryza sativa, 29 from Brassica rapa, 23 from Brassica oleracea and 65 from Brassica napus. The JmjC domain proteins can be grouped into7 groups based on the phylogenetic tree and domain organization. Different colors show different groups. JmjC domain protein sequences were aligned using ClustalW, and the phylogenetic tree analysis was performed using 
MEGA7.0.The trees were constructed with the following settings: tree inference as neighbor-joining; include sites as pairwise deletion option for total sequences analysis; substitution model as p-distance.

A

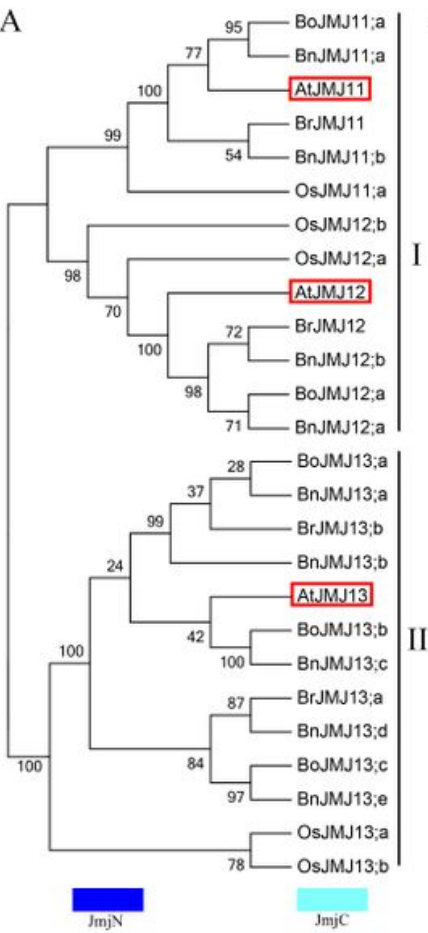

B

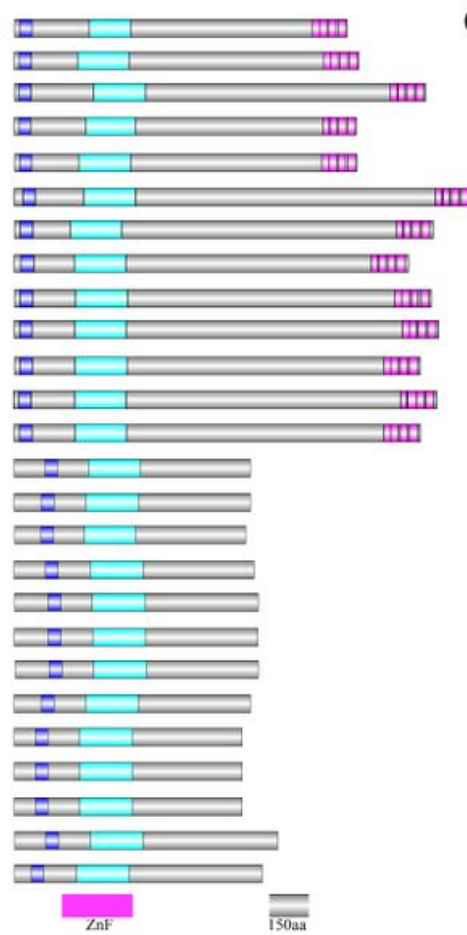

$\mathrm{C}$

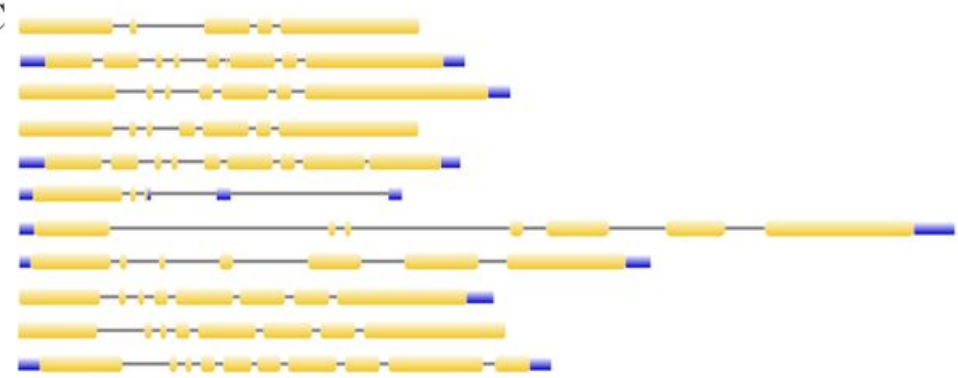

$\square+$

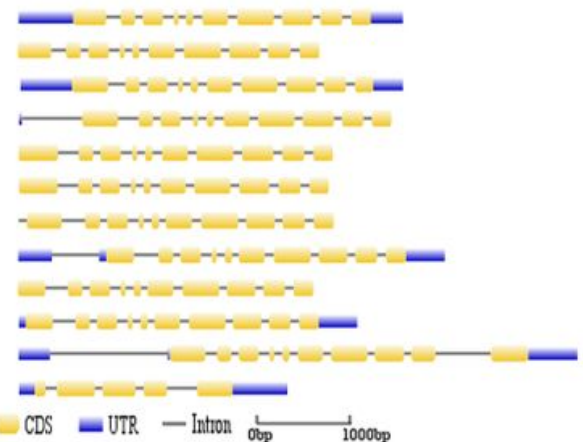

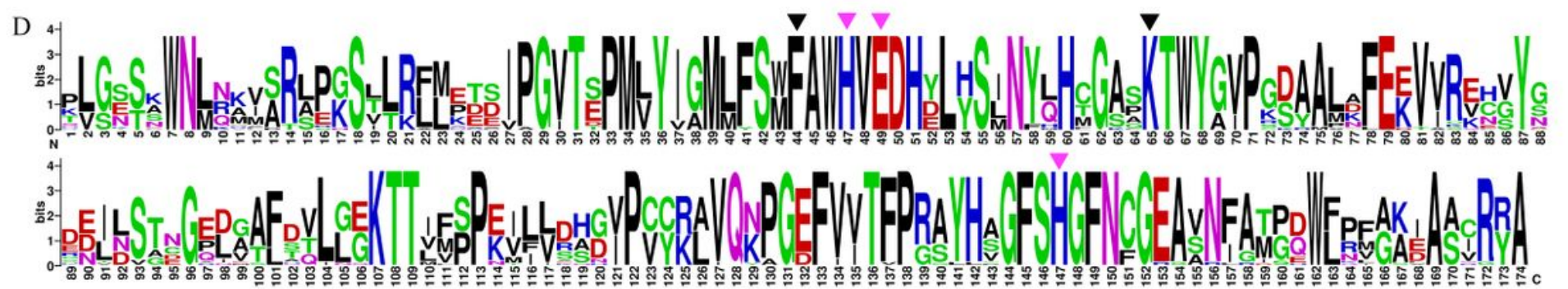

Figure 3

The schematic diagrams of Group-KDM4/JHDM3 A. Phylogeny tree, B. domain organization, C. Gene structure, D. Logos analysis of JmjC domain. 


\section{Group-KDM5A}

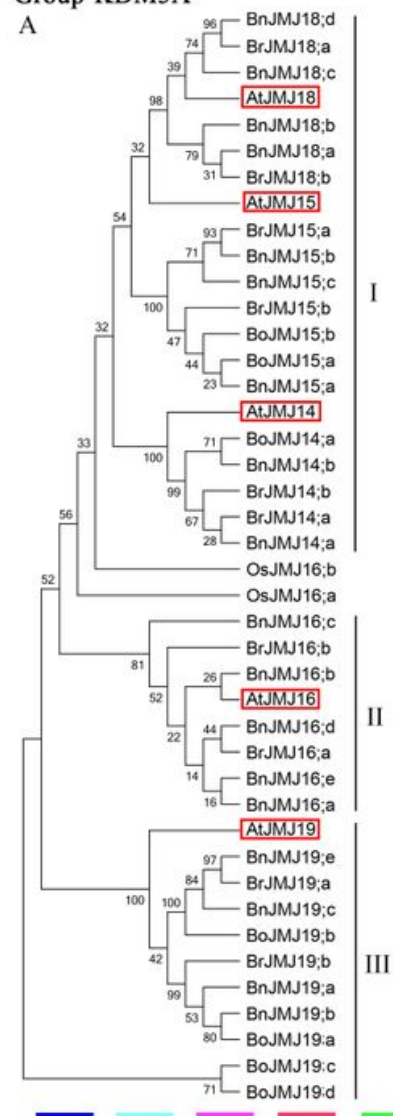

$\mathrm{B} \equiv$

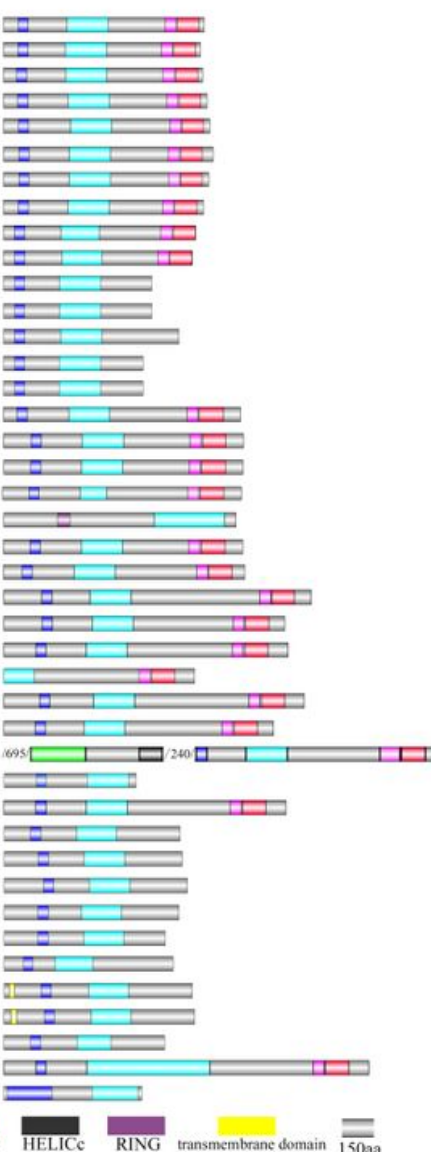

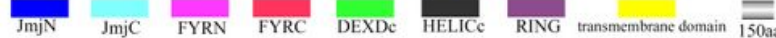

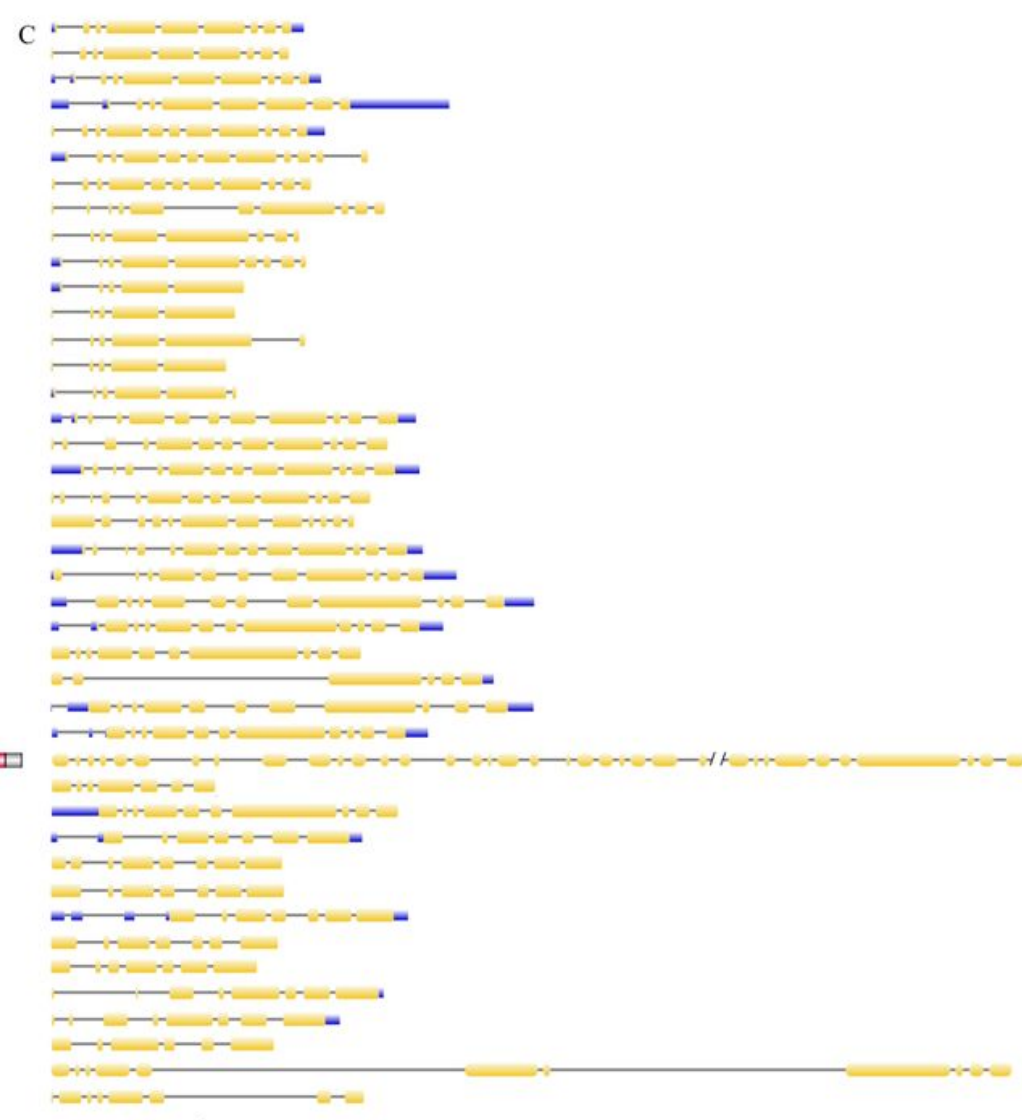

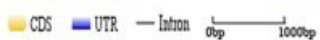

D
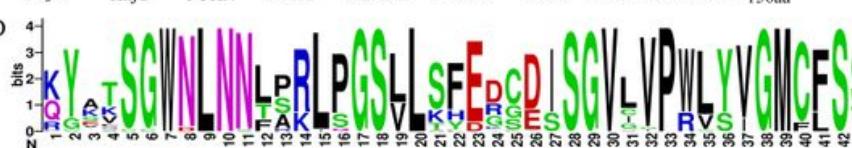

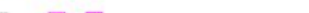

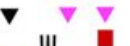

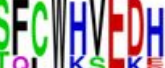
等
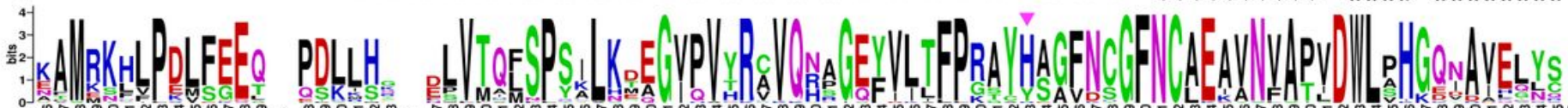

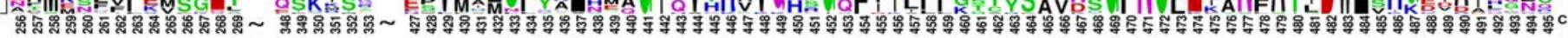
Group-KDM5B
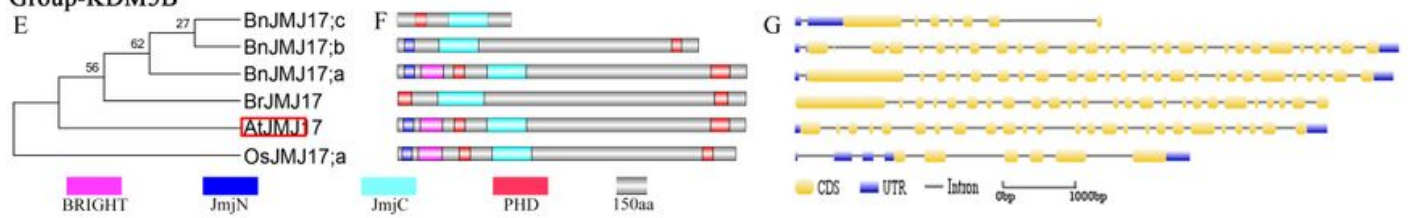

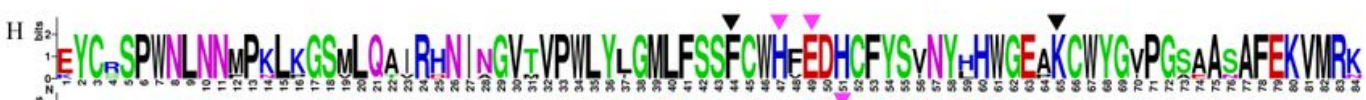

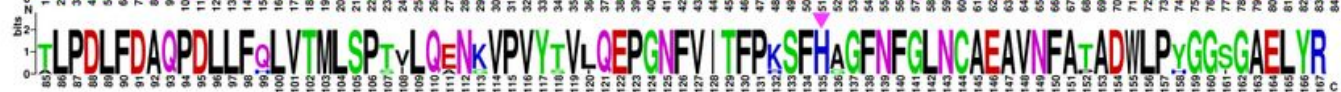

\section{Figure 4}

The schematic diagrams of Group-KDM5A/B A/E. Phylogeny tree, B/F. domain organization, C/G. Gene structure, D/H. Logos analysis of JmjC domain. 
Group-JmjC domain-only A

A

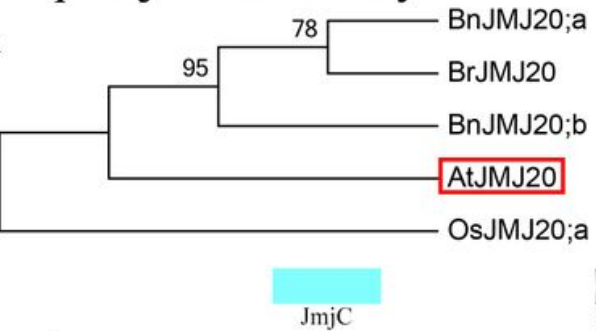

B

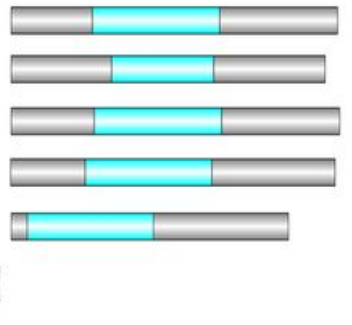

$\mathrm{C}$

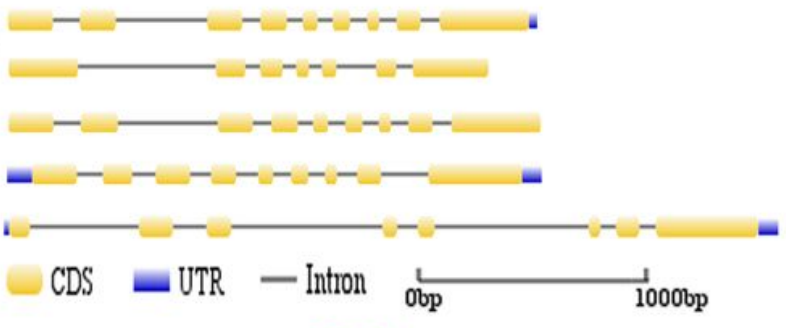

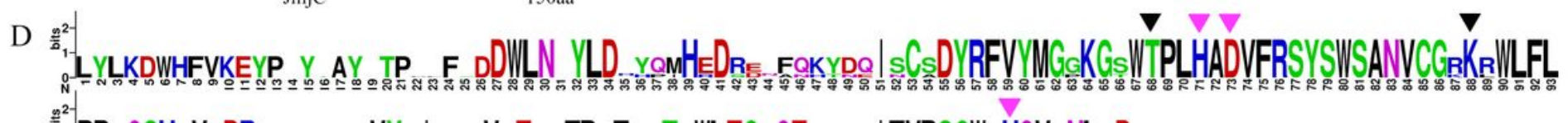

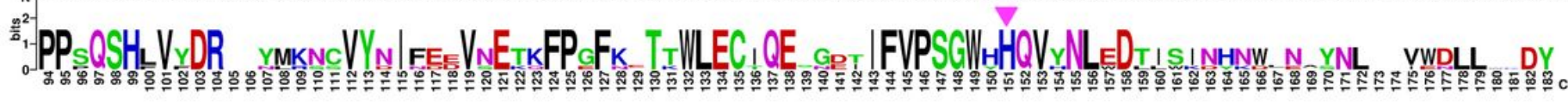

Group-JmjC domain-only B

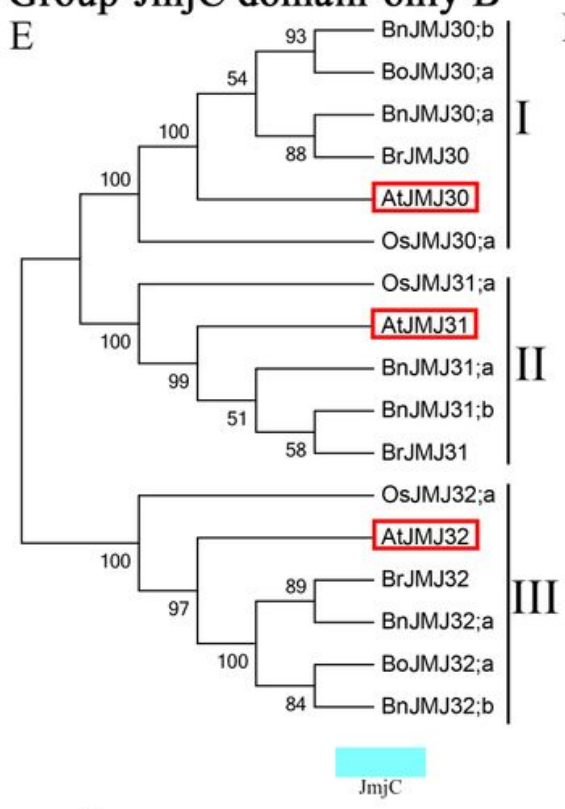

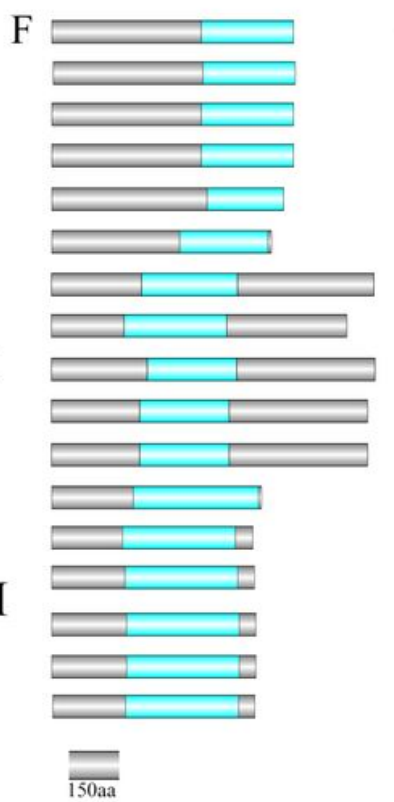

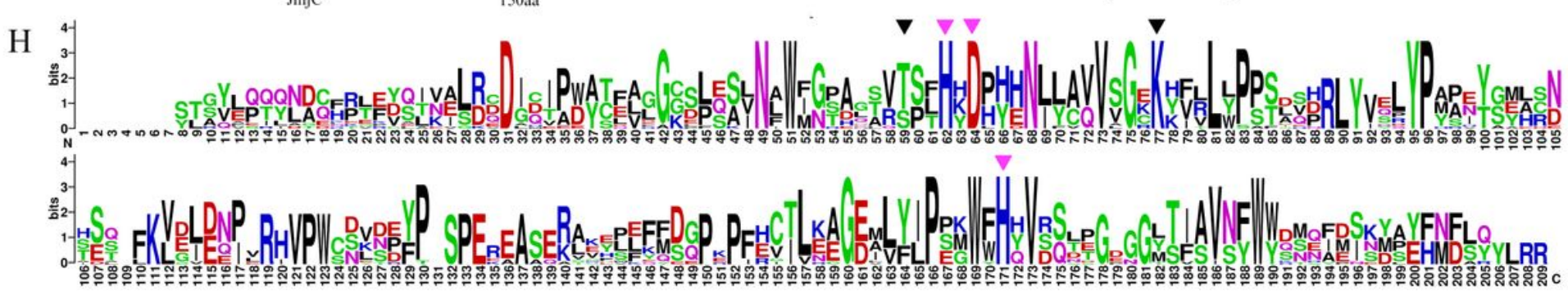

\section{Figure 5}

he schematic diagrams of Group-JmjC domain-onlyA/B A/E. Phylogeny tree, B/F. domain organization, C/G. Gene structure, D/H. Logos analysis of JmjC domain. 

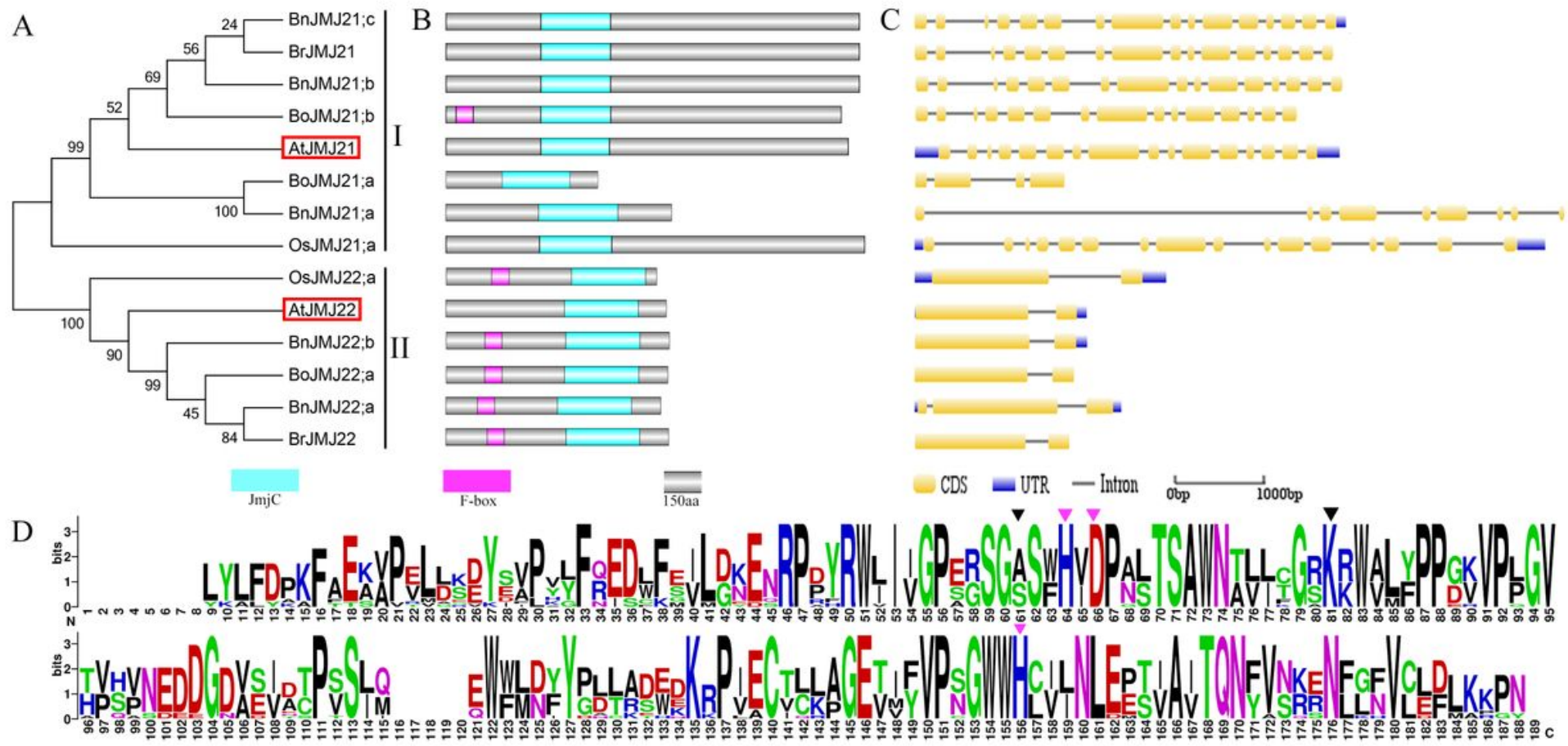

Figure 6

The schematic diagrams of Group-JMJD6 A. Phylogeny tree, B. domain organization, C. Gene structure, D. Logos analysis of JmjC domain. 
A

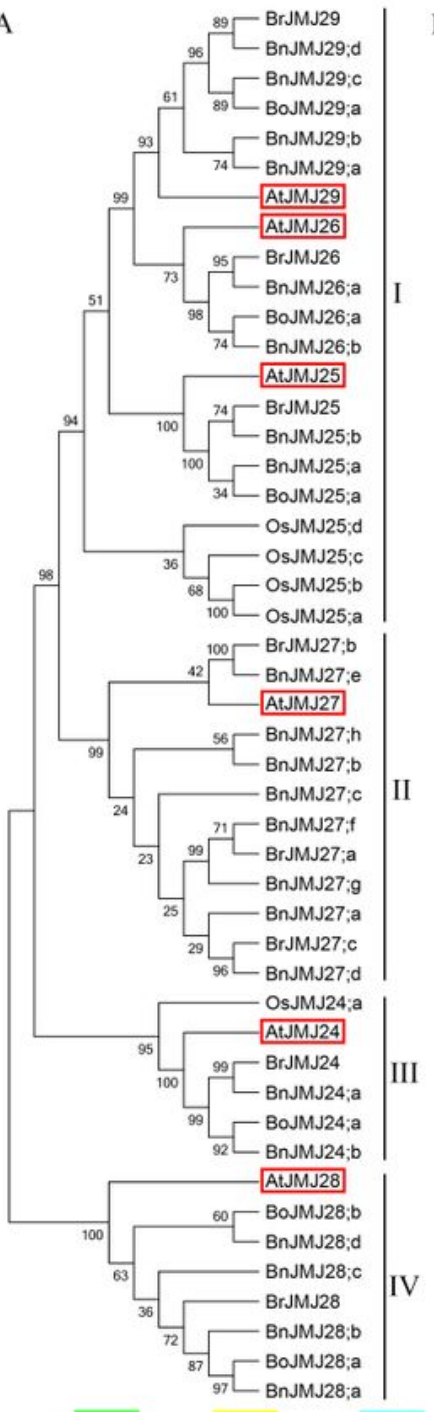

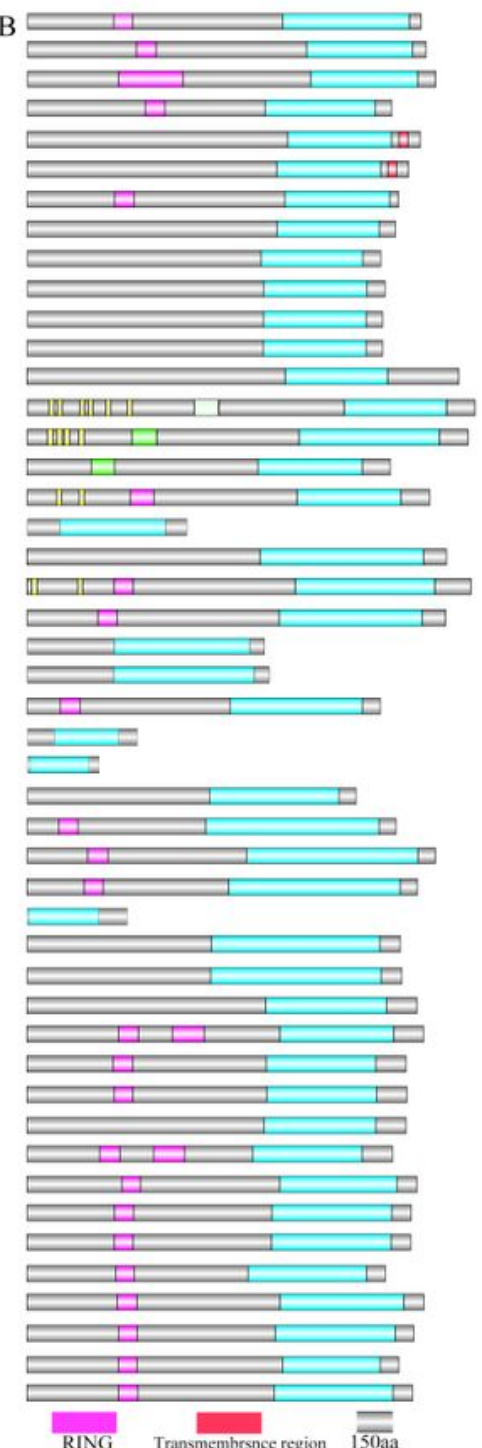

$\mathrm{C}$

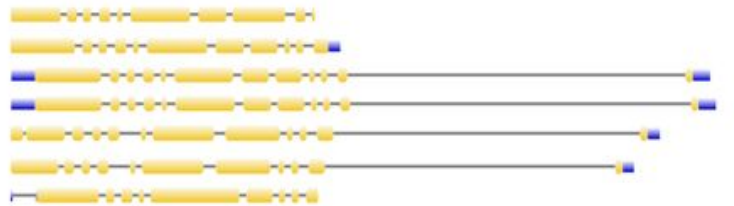

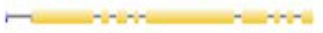

-

- - -

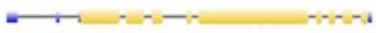

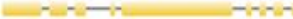

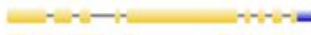

(m) -

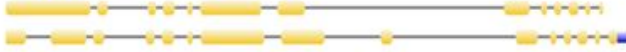

(-n-n- - -

- - - -

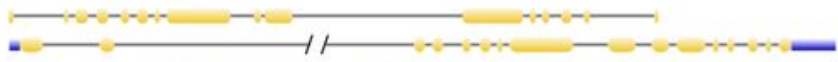

-

-

(n)

$-\cdots+\cdots$

- - -

n-

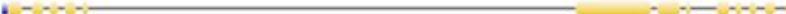

Ham

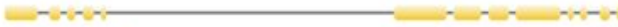

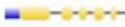

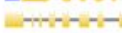

$-$

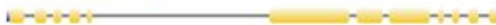

-

-

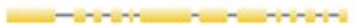

n-...

-

-

-

n-w - n

- $\ldots-\ldots \ldots$

ㄴ.m …

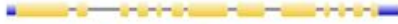

-

-

CDS $=$ UTR - Intron oep looobp

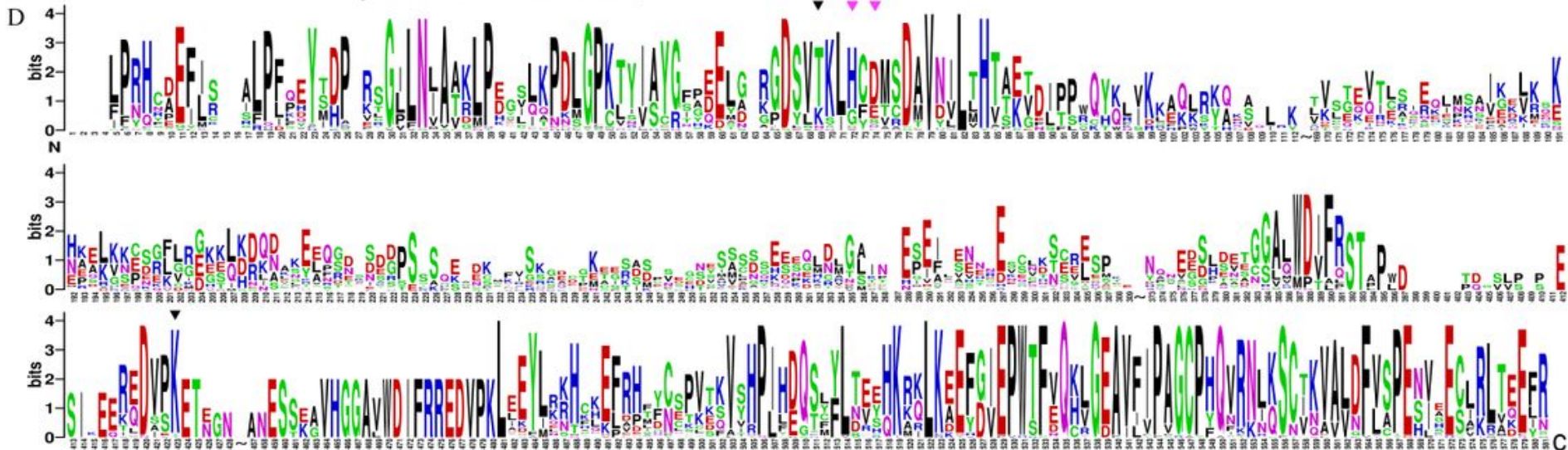

\section{Figure 7}

The schematic diagrams of Group- KDM3\&JHDM2 A. Phylogeny tree, B. domain organization, C. Gene structure, D. Logos analysis of JmjC domain. 


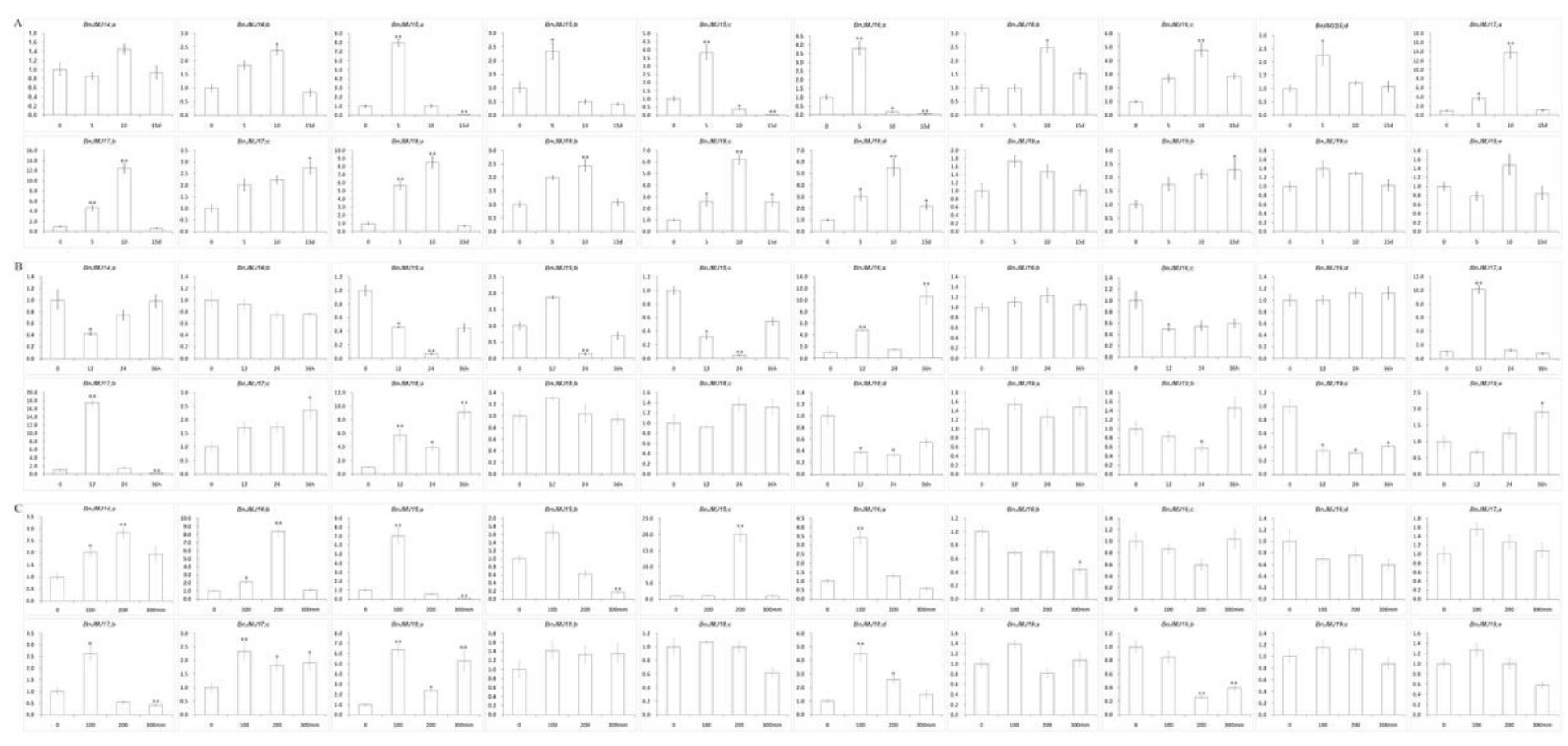

Figure 8

Expression of B. naups KDM5 subfamily in response to drought, high temperature or $\mathrm{NaCl}$ stresses Many of BnJMJ14-19 genes involved in drought, high temperature or $\mathrm{NaCl}$ stress response. The error bars depict SD, an asterisk represent corresponding gene significantly up- or down-regulated by Student's t test between the treatment and the control $(0.01<P<0.05)$, two represent $(p<0.01)$.

\section{Supplementary Files}

This is a list of supplementary files associated with this preprint. Click to download.

- Additionalfile3NJphylogenetictreeofKDM5.jpg

- Additionalfile1DetailsofJmjCgenesinBrassica.xlsx

- Additionalfile2JmjCgenesduplicatedgenesofB.rapa.xlsx

- Additionalfile4PrimerslistofgroupVinB.napus.xlsx 